\title{
Delineating spatiotemporal and hierarchical development of human fetal innate lymphoid cells
}

\author{
Chen Liu ${ }^{1,7}$, Yandong Gong ${ }^{2,7}$, Han Zhang ${ }^{3,7}$, Hua Yang ${ }^{4,7}$, Yang Zeng ${ }^{2}$, Zhilei Bian (iD ${ }^{5}$, Qian Xin ${ }^{1}$, Zhijie Bai iD ${ }^{1}$, Man Zhang ${ }^{1}$,

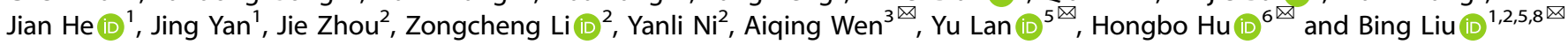

(c) The Author(s) 2021

\begin{abstract}
Whereas the critical roles of innate lymphoid cells (ILCs) in adult are increasingly appreciated, their developmental hierarchy in early human fetus remains largely elusive. In this study, we sorted human hematopoietic stem/progenitor cells, lymphoid progenitors, putative ILC progenitor/precursors and mature ILCs in the fetal hematopoietic, lymphoid and non-lymphoid tissues, from 8 to 12 post-conception weeks, for single-cell RNA-sequencing, followed by computational analysis and functional validation at bulk and single-cell levels. We delineated the early phase of ILC lineage commitment from hematopoietic stem/progenitor cells, which mainly occurred in fetal liver and intestine. We further unveiled interleukin-3 receptor as a surface marker for the lymphoid progenitors in fetal liver with T, B, ILC and myeloid potentials, while IL-3RA- lymphoid progenitors were predominantly B-lineage committed. Notably, we determined the heterogeneity and tissue distribution of each ILC subpopulation, revealing the proliferating characteristics shared by the precursors of each ILC subtype. Additionally, a novel unconventional ILC2 subpopulation (CRTH2 $\mathrm{CCR9}^{+}$ILC2) was identified in fetal thymus. Taken together, our study illuminates the precise cellular and molecular features underlying the stepwise formation of human fetal ILC hierarchy with remarkable spatiotemporal heterogeneity.
\end{abstract}

Cell Research (2021) 31:1106-1122; https://doi.org/10.1038/s41422-021-00529-2

\section{INTRODUCTION}

Innate lymphoid cells (ILCs), lacking expression of known immune lineage markers and antigen-specific receptors, are a family of lymphocytes as innate equivalents of T cells, and play critical roles in immune response and tissue homeostasis. ${ }^{1-6}$ ILCs have been originally categorized into three, and now into five groups, namely, nature killer (NK) cell, ILC1, ILC2, ILC3 and lymphoid tissueinducer (LTi) cell. ${ }^{2,7-12}$ Both ILCs and T cells are proposed to be derived from lymphoid progenitors like common lymphoid progenitors (CLPs) and lymphoid-myeloid primed progenitors (LMPPs) in human and mouse, followed by the stepwise commitment to each lymphocyte fate. ${ }^{13-18}$ Commitment to ILC is accompanied by the loss of T cell, B cell and dendritic cell (DC) potentials. ${ }^{15,19-22}$ The final step of ILC maturation is proposed to occur in the tissues where ILCs are located. ${ }^{23,24}$ Transcriptional regulation along ILC development in mouse and human is relatively conservative, involving more than a series of transcription factors (TF). Specifically, ID2, NFIL3, PLZF, TCF1, GATA3, ETS1, TOX, T-BET, EOMES, RORa, BCL11B, AHR and RORyt have been proved to drive commitment, specification and maturation of ILCs from lymphoid progenitors. ${ }^{19,25-35}$

In humans, the ILC progenitors (ILCPs) in circulation are ILCcommitted precursors expressing ID2, TOX and ZBTB16. They are identified as $\mathrm{Lin}^{-} \mathrm{CD} 45^{+} \mathrm{CD} 7^{+} \mathrm{CD} 127^{+} \mathrm{CD} 117^{+} \mathrm{CRTH} 2^{-}$phenotype, subsets of which have the capacity to generate all the ILC subsets in vitro and in vivo. ${ }^{36}$ Intriguingly, the ILCPs from human fetal liver predominantly generate ILC3s in co-culture system, suggestive of their remarkable heterogeneity and dynamic spatiotemporal lineage potentials. ${ }^{36,37}$ These precursors might be derived from $\mathrm{CD} 34^{+} \mathrm{CD} 117^{+} \mathrm{ROR}^{+}$progenitors that also have been found in the second lymphoid tissues. The ILCPs expressing of KLRG1, NKp46 and CD56 were reported to possess different ILC lineage preferences in peripheral blood (PB) and tonsil. ${ }^{21,38,39}$ While some intermediate ILC progenitors/precursors have been described, ${ }^{36,40,41}$ the unbiased hierarchical investigation of ILC differentiation process in the hematopoietic, lymphoid and nonlymphoid tissues in early human development is still unclear. For instance, the intermediate progenitors/precursors along ILC development that bridge hematopoietic stem and progenitors (HSPCs) and committed ILC progenitors remain elusive.

Spatial-temporal distribution and tissue-specific transcriptional heterogeneity of ILCs at single-cell resolution in adult human tissues have been revealed in recent years while those in human fetal tissues remain poorly characterized. ${ }^{42-45}$ Thymus, the primary lymphoid tissue for $T$ cell production, has been recently reported to contain different subsets of ILCs. ${ }^{46-48}$ The LTi-like ILCs, which are important for the development of functional medulla thymic epithelial cells, are readily detectable in human fetal thymus. ${ }^{49,50}$

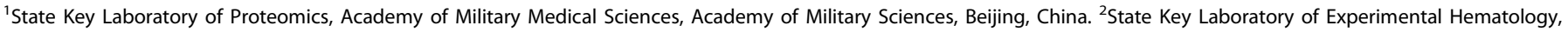

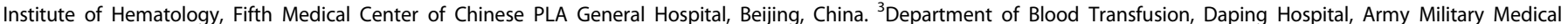

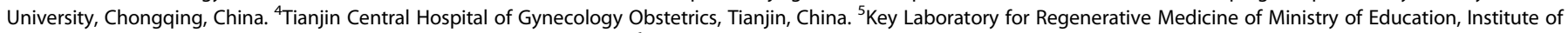

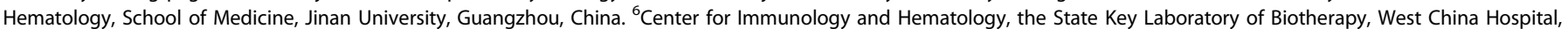

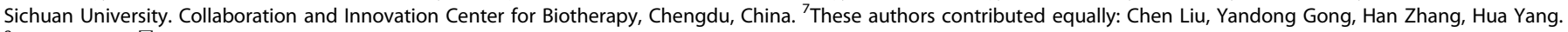
${ }^{8}$ Lead contact. ${ }^{凶}$ email: dpyysxkwaq@hotmail.com; rainyblue_1999@126.com; hongbohu@scu.edu.cn; bingliu17@yahoo.com
} 
Importantly, the ILC2-like cells with T cell characteristics are identified in both mouse and human fetal thymus by single-cell RNA-sequencing (scRNA-seq). ${ }^{46,47}$ Consistent with the observation of intrathymic ILC2 development, ${ }^{51-54}$ ILC2 becomes the major ILC subset in the thymus of adult mice. ${ }^{50}$ All these findings pinpoint the potential importance of ILC2 in thymus development and homeostasis.

Here, we provided an unprecedented single-cell transcriptome atlas of stepwise ILC ontogeny in early human fetus using cells from fetal hematopoietic, lymphoid and non-lymphoid tissues spanning 8 to 12 post-conception weeks (PCW). We identified a series of intermediate cell populations along the differentiation paths from human HSPCs to mature ILCs, especially the interleukin-3 receptor alpha positive $\left(\mathrm{IL}_{-} 3 \mathrm{RA} \mathrm{A}^{+}\right.$) lymphoid progenitors with $\mathrm{T}, \mathrm{B}, \mathrm{ILC}$ and myeloid potentials in fetal liver, combined with computational prediction and functional validation at bulk and single-cell levels. Our study revealed dynamic changes of cell cycle status along with ILC development, from ILC progenitors (quiescent) to the precursors of each ILC subtype (proliferative) and to mature ILCs (quiescent), as well as the heterogeneity and tissue distribution of ILCs. Taken together, our study illuminated the precise molecular events underlying human ILC development at early fetal stage.

\section{RESULTS}

The spatio-temporal distribution of ILCs in human fetal organs The development of human ILCs at fetal stage remains to be defined. It has been postulated that they might be from HSPCderived lymphoid progenitors, which migrate into lymphoid and non-lymphoid tissues for final maturation. ${ }^{37}$ To investigate the molecular basis underlying the human ILC development path from HSPCs to lymphoid progenitors and to each type of ILCs in different tissues at single-cell resolution, we preformed scRNA-seq using cells isolated from the human fetal hematopoietic (liver), lymphoid (thymus and spleen) and non-lymphoid (intestine, skin and lung) tissues at 8,10 and 12 PCW that are the critical timeframes for ILC development and initiation of lymphoid organogenesis (Fig. 1a; Supplementary information, Fig. S1). The spatiotemporal distribution of mature ILCs and upstream HSPCs and lymphoid progenitors in the fetal tissues were firstly examined by flow-cytometry based on the surface markers indicated in Fig. 1b, c and Fig. 1d, e, respectively. To further confirm the identities of three types of helper ILCs, flow cytometry analysis with co-staining for lineage-specific transcription factors including T-bet, GATA3 and RORyt was conducted (Supplementary information, Fig. S2a). Among $\mathrm{Lin}^{-} \mathrm{CD} 45^{+} \mathrm{CD} 127^{+} \mathrm{ILCs}, \mathrm{CRTH} 2^{+}$ and $\mathrm{CD} 117^{+}$ILCs showed the highest expression level of GATA3 and RORyt, indicating their conventional ILC2 and ILC3 identities, respectively. As expected, the highest expression of T-bet was detected in $\mathrm{Lin}^{-} \mathrm{CD} 45^{+} \mathrm{CD} 127^{-} \mathrm{CD} 56^{+}$cells, suggestive of killer ILCs. The $\mathrm{CRTH}^{-} \mathrm{CD} 117^{-}$ILCs were annotated as "putative ILC1 cells", based on the lack of conventional CD127 $7^{+}$ILC1specific markers. ${ }^{1}$ These cells showed heterogeneous expression of T-bet, GATA3 and RORyt, suggesting that they were heterogeneous, containing classical ILC1s and non-classical ILC2s and ILC3s. Within lineage (CD3, CD4, CD5, FceRI, CD11c, CD11b, CD14 and CD19)-negative ( $\left.\mathrm{Lin}^{-}\right) \mathrm{CD} 34^{-} \mathrm{CD} 45^{+}$cells, $\mathrm{CD} 56^{+} \mathrm{CD} 127^{-}$cytotoxic ILCs, and $\mathrm{CD} 56^{-} \mathrm{CD} 127^{+}$helper ILCs that were further divided

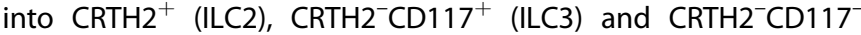
(putative ILC1) were found in all tested tissues (Fig. 1c; Supplementary information, Fig. S2b). The percentages of putative ILC1 and ILC2 in thymus were much higher than those in other tissues. Intriguingly, ILC3 was the dominate population in all tissues except for thymus, accounting for over $90 \%$ of $\mathrm{Lin}^{-} \mathrm{CD} 45^{+} \mathrm{CD} 127^{+}$ ILCs (Fig. 1c). The absolute cell numbers of putative ILC1 and ILC2 in thymus were also higher than those in other tissues except for intestine (Supplementary information, Table S1). In addition, most, if not all, CRTH2 ${ }^{+}$ILC2 in skin were CD117 positive, which was different from those in other tissues (Supplementary information, Fig. S2b). NKp44, the important surface marker of ILC3, ${ }^{10}$ became detectable around week 12, mostly restricted to ILC3 in intestinal and lung, which was consistent with previous studies that the majority of ILC in the fetal small intestines express both CD69 and NKp44 from week 12 onward and ILC3s in fetal livers at 6 to 20 PCW were mainly NKp44 ${ }^{-55-57}$ (Supplementary information, Fig. $\mathrm{S} 2 \mathrm{c}$ ).

As expected, fetal liver, the predominant organ of fetal hematopoiesis at the range of our study, ${ }^{58}$ had the largest proportion of HSPCs $\left(\mathrm{Lin}^{-} \mathrm{CD} 45^{+} \mathrm{CD} 34^{+} \mathrm{CD} 127^{-}\right)$(Fig. 1d, e; Supplementary information, Fig. S3a, b). The percentage of lymphoid progenitors ( $\mathrm{Lin}^{-} \mathrm{CD} 45^{+} \mathrm{CD} 34^{+} \mathrm{CD} 127^{+}$) in fetal thymus was the highest among all the examined tissues, which presumably contained T/ILC progenitors. ${ }^{46,51,53,59-62}$ Meanwhile, our data showed that fetal intestine had a considerable proportion of lymphoid progenitor at week 8, which was consistent with the previous studies indicating that intestine is an ILC-rich organ at fetal and adult stages ${ }^{23,63}$ (Fig. 1e; Supplementary information, Fig. $\mathrm{S} 3 \mathrm{a}, \mathrm{b})$. The percentage of lymphoid progenitors in intestine reduced dramatically at $12 \mathrm{PCW}$, correlated with the increased $\mathrm{CD} 161^{+} \mathrm{CD} 127^{+/-}$ILCs, presumably due to the formation of mesenteric lymph nodes ( $\mathrm{mLNs}$ ) in the beginning of the second trimester of pregnancy. ${ }^{8}$ Interestingly, the frequency of $\mathrm{CD} 161^{+} \mathrm{CD} 127^{+}$ILCs, mostly representing the helper ILCS, dramatically reduced in thymus from 8 to 12 PCW $(20.7 \%$ to $1.98 \%$, of Lin $^{-} \mathrm{CD} 45^{+} \mathrm{CD} 34^{-}$cells) (Fig. 1e; Supplementary information, Fig. S3a, b), which was in accordance with the dynamics of ILC in the previous studies. ${ }^{46}$ Thus, our study reveals the spatiotemporal distribution of lymphoid progenitor and ILCs in human fetal organs.

After confirming the presence of mature ILC and potent upstream progenitor or precursor cells in hematopoietic, lymphoid and non-lymphoid tissues, we labeled the cells from each tissue of individual embryo with hashtag oligos (HTO) before fluorescence-activated cell sorting (FACS) analysis. Within $\mathrm{Lin}^{-} \mathrm{CD} 45^{+}$cells, HSPCs $\left(\mathrm{CD} 34^{+} \mathrm{CD} 127^{-}\right)$, lymphoid progenitors $\left(\mathrm{CD} 34^{+} \mathrm{CD} 127^{+}\right), \mathrm{CD} 161^{+} \mathrm{ILCs}\left(\mathrm{CD} 34^{-} \mathrm{CD} 127^{+/-} \mathrm{CD} 161^{+}\right)$as well as $\mathrm{CD}^{-} \mathrm{CD} 161^{-}$cells were sorted and mixed in the ratios indicated in Supplementary information, Fig. S1. To avoid genetic bias, two embryos were used for each time point. Cells from tissues were then pooled for $10 \times$ genomics scRNA-seq (Fig. 1a). After quality control and doublets exclusion, a total of 31,233 cells were captured, including 9477 cells from liver, 5105 cells from intestine, 4220 cells from thymus, 2112 cells from spleen, 4868 cells from skin and 5451 cells from lung (Supplementary information, Fig. $\mathrm{S} 4 \mathrm{a}-\mathrm{c}$ ). The average number of genes and transcripts per cell was 2,837 and 12,982 , respectively (Supplementary information, Fig. S4d, e).

\section{Single-cell transcriptomic profiling of lymphoid-related populations in human fetus}

To facilitate the delineation of lymphoid-related populations, we removed the unrelated hematopoietic lineages such as granulocyte-macrophage progenitors, megakaryocyte-erythroid progenitors, and their progeny cells, together with the contaminated non-hematopoietic cells, including epithelial, endothelial and mesenchymal cells (Supplementary information, Fig. S5a, d and Table S2). For the same purpose, only the most primitive $B$ cell progenitors (pre pro-B cells), and T cell progenitors (early thymic progenitor, ETP) were included for further analyses (Supplementary information, Fig. S5a-e). We identified ten lymphoid-related clusters according to the expression of curated genes (Fig. 2a, b; Supplementary information, Table S3). HSPC cluster was distinguished by the enrichment of stem and progenitor cells-related genes, such as CD34, MYCN and MECOM. Two lymphoid progenitor (LP) clusters, LP1 and LP2, were readily identified based on the expression of lymphoid-specific genes CD7 and IL7R. The 
a

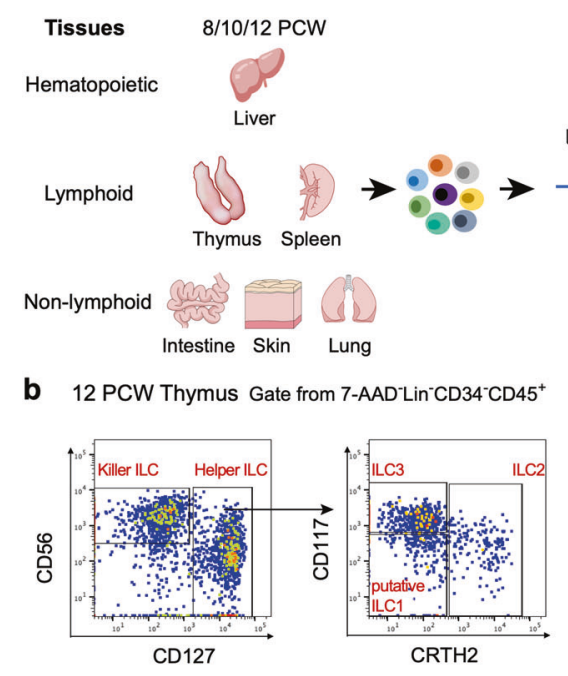

Gate from 7-AAD-Lin-CD45+
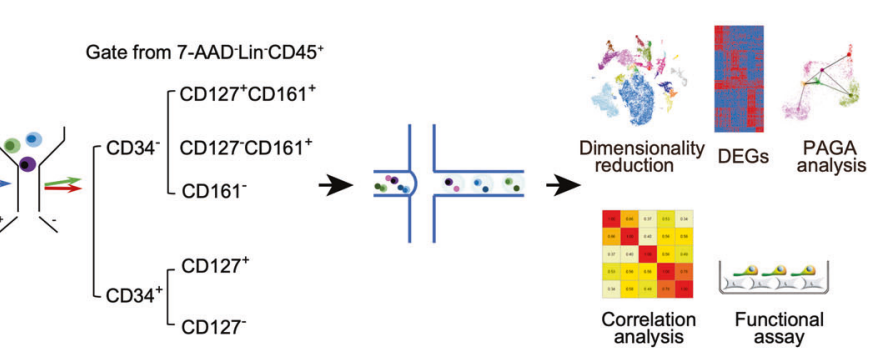

d Gate from 7-AAD-
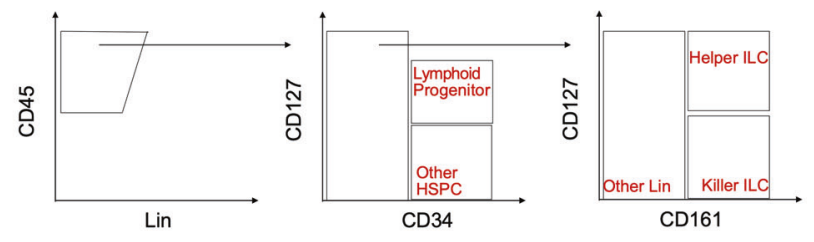

c

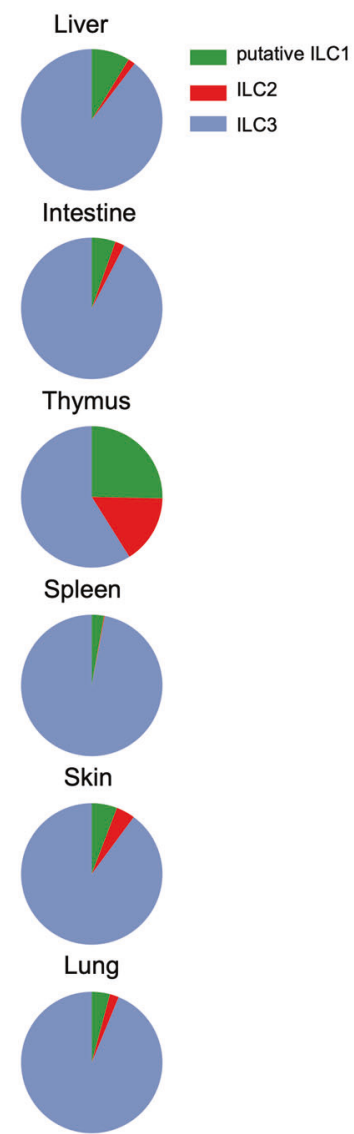

e

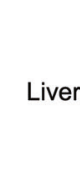

Intestine

Thymus

Spleen

Lung

\section{PCW}

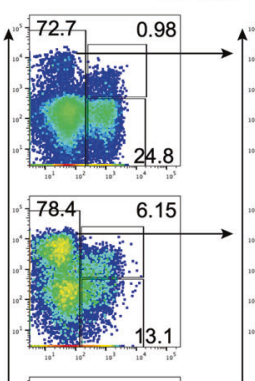

$92.6 \quad 2.07$
$12 \mathrm{PCW}$

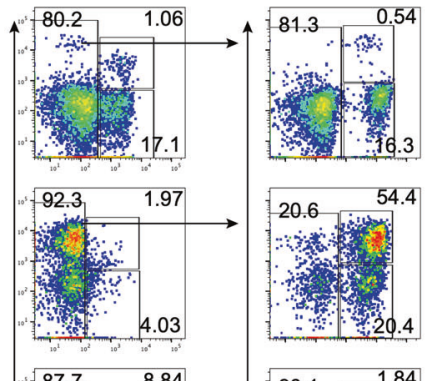

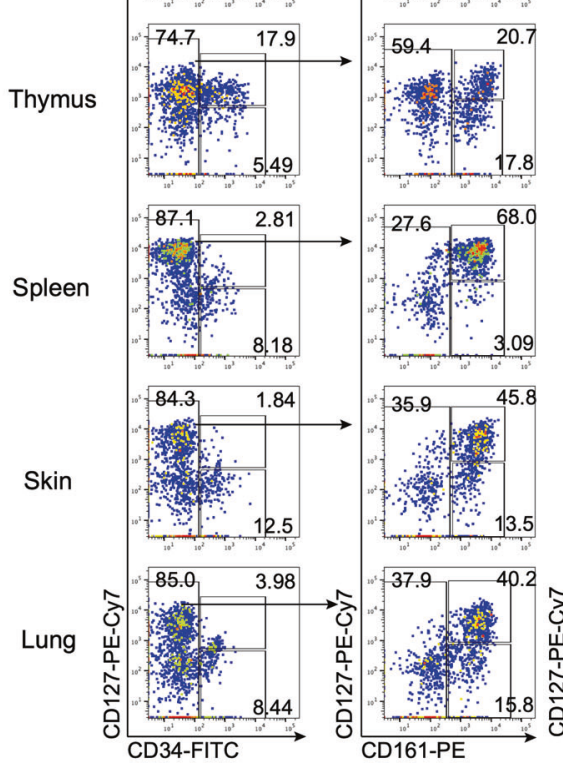
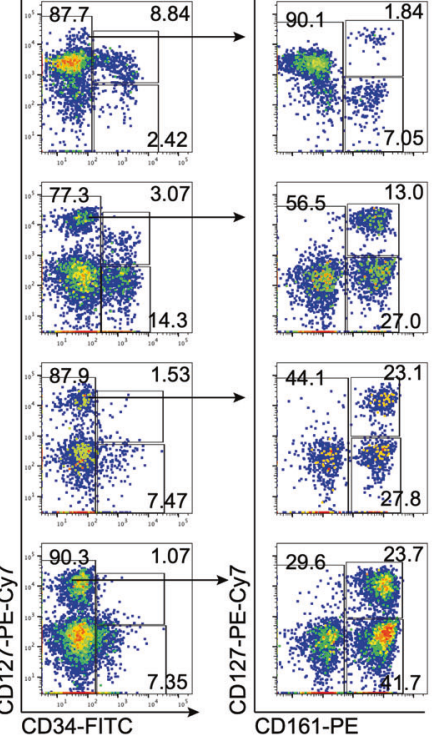

expression level of CD34 in LP1 was higher than in LP2, suggesting that LP1 might be upstream of LP2. In addition to CD7 and IL7R, Blineage genes $C D 79 A$ and $C D 79 B$ were highly expressed in pre pro$B$ cells. Meanwhile, enrichment of GATA3 and TCF7 in ETP cluster was in line with its T-lineage potential. Precursors of plasmacytoid dendritic cells (pre $\mathrm{pDC}$ ) were characterized by the expression of IRF7, CLEC4C and remaining expression of CD34.

IL7R and CD7 were also highly expressed in ILCPs and three ILC populations (Group 1, 2, 3 ILC), consistent with the flow cytometry results. ILCPs expressed low level of CD34, indicative of their progenitor identity, and the expression of KLRB1 (encoding CD161) was also lower than mature ILCs (Fig. 2b). Importantly, $R O R C$, encoding the essential TF (RORyt) for all ILCs and especially ILC3 development, ${ }^{21,30}$ was enriched in ILCP cluster. Consistent with the expression of cell surface marker, Group 1 ILC showed lower expression of IL7R and KIT, but higher expression of KLRB1, than other ILC groups. The identification of Group 1 ILC was also confirmed by the enriched EOMES, which encodes the signature TF for cytotoxic ILC, and IFNG (Fig. 2b, c). Group 2 ILC cluster was annotated based on the expression of GATA3 and PTGDR2 
Fig. 1 Study strategy and ILC-related populations analysis overview. a Schematic overview of the strategy of this study. Cells from human fetal hematopoietic (liver), lymphoid (thymus and spleen) and non-lymphoid (intestine, skin and lung) tissues at indicated gestational stages were first labeled with cell hashing antibodies and fluorescence-labeled antibody for sorting based on the sorting strategy in e. The cells from 2-6 tissues were pooled and then loaded on a droplet-based 10x genomics scRNA-seq platform. Cells of each tissue from different samples were distinguished by cell hashing barcode. The information of individual sample was listed in Supplementary information, Fig. S1. Two replicates per developmental stage, with a total of 6 samples were used in this study. $\mathbf{b}$ The gating strategy to identify human mature ILCs with example from 12 PCW thymus. In the 7AAD lineage (CD3, CD4, CD5, FceRI, CD11 c, CD11b, CD14 and CD19)-negative (Lin $\left.{ }^{-}\right) C^{-} 34{ }^{-} C^{-}$45 $5^{+}$ cells, there are $\mathrm{CD} 56^{+} \mathrm{CD} 127^{-}$killer ILCs and $\mathrm{CD} 56^{-} \mathrm{CD} 127^{+}$helper ILC subsets. The helper ILCs are divided into CRTH2 ${ }^{+}$ILC2, $\mathrm{CRTH} 2^{-} \mathrm{CD} 117^{+}$ ILC3 and CRTH2 ${ }^{-} \mathrm{Kit}^{-}$putative ILC1. c Pie charts show the proportions of three mature helper ILCs of each organ based on flow cytometry analysis. The proportions were calculated based on more than three independent experiments. d Sorting strategy of human fetal lymphoid progenitors and ILC-associated populations. In the 7-AAD ${ }^{-}$Lin $^{-} \mathrm{CD} 45^{+}$cells, $\mathrm{CD} 34^{+} \mathrm{CD} 127^{+}$lymphoid progenitors, CD34 ${ }^{+} \mathrm{CD} 127^{-} \mathrm{HSPCs}$, $\mathrm{CD} 34^{-} \mathrm{CD} 161^{+} \mathrm{CD} 127^{+/-} \mathrm{ILCs}$ and $\mathrm{CD} 34^{-} \mathrm{CD} 161^{-}$cells were sorted from each tissue, and mixed at the ratios indicated in Supplementary information, Fig. S1 for scRNA-seq. e The flow cytometry results show that the percentages of the cell populations identified in d in each tissue change from 8 to 12 PCW. The results are representative of 2-3 independent experiments in each gestational week.

(encoding CRTH2). ${ }^{64}$ Group 3 ILC was featured by the expression of $R O R C$, and the highest expression level of IL7R and KIT. Hierarchical clustering analysis showed that LP2 and ILCP were closely located (Fig. 2d), indicating a strong correlation between these populations. Group 1 ILC, consisting of NK cells, were separated from Group 2 ILC and Group 3 ILC, suggesting the developmental branch of cytotoxic and helper ILCs as discovered in mouse ${ }^{65-67}$ (Fig. 2d).

The spatial distribution of these cells revealed by the scRNA-seq data was largely in line with the flow cytometry results. The majority of HSPCs were located in fetal liver, meanwhile fetal liver also contained two types of LPs and pre pro-B cells, reconciling with its function as the niche for early development of lymphoid progenitors and B cells. Notably, the proportion of LP1 was higher than that of LP2 in fetal liver, but more LP2s and ILCPs were found in intestine, collectively suggesting that intestine as the ILCs-rich organ which might provide the optimized microenvironment for ILCs development (Fig. 2d, e; Supplementary information, Fig. S5f). The majority of ETPs resided in thymus. $41 \%$ of ILCs in thymus were ILC2, which was consistent with the flow cytometry data (Fig. 1b, c; Supplementary information, Fig. S2b). Taken together, our scRNA-seq data revealed the cellularity and tissue distribution of HSPCs, lymphoid progenitors and ILCs at the end of the first trimester of pregnancy.

\section{The IL-3RA as the marker of lymphoid progenitors with T, B, ILC and myeloid potential in human fetal liver}

To delineate the lymphocyte lineage differentiation in human fetal liver at early stage, the progenitor populations identified above were selected for further analysis. Partition-based graph abstraction (PAGA) analysis was used to demonstrate their topological location of developmental trajectories, revealing their putative developmental orientation (Fig. 3a, b). The result showed HSPC was at the start point, directly followed by LP1, accompanied by the downregulation of stem and progenitorrelated TF-coding genes, like CEBPA, ERG, HLF, MECOM and $S P I 1^{68}$ (Fig. $3 a-c)$. Consistently, few HSPCs displayed a lineage-primed state predicted by STEMNET analysis (Supplementary information, Fig. S6a, b and Table S4). On the other hand, the lymphoid lineage (including T, B and ILC cells) potentials of LP1 was predicted, although the majority of LP1s were still un-committed since these cells were located in the central position of the STEMNET map. CEBPA and MECOM were further downregulated in LP2s (Fig. 3c). LP2s were likely downstream of LP1 based on the PAGA results, and they might have divergent development paths, including myeloblast and pDC (mature pDC: GSE143002 ${ }^{69}$ ) paths as well as T, B and ILC paths (Fig. 3b; Supplementary information, Fig. S6a, b). More importantly, NFIL3, transitionally expressed before ID2 that is important for ILC commitment, was initially upregulated in LP2 and sustained its expression in ILCP. ${ }^{27}$ (Fig. 3c). ILCP, showing the strongest connection with LP2 (Fig. 3b), was featured by the enrichment of ILC specification- related genes, such as RORC and EOMES. High expression of $R U N X 3$ in ILCPs suggested their differentiation potentials to both cytotoxic and helper ILCs especially ILC3. ${ }^{70,71}$ As anticipated, the pre pro- $B$ were restricted to $B$-cell lineage, with upregulation of $B$ cell feature TFs-encoding genes including EBF1 and PAX5. ETP, highly expressing GATA3, HES1 and MYB, showed both T and ILC potentials, which was consistent with the previous findings showing that the uncommitted $\mathrm{Lin}^{-} \mathrm{CD} 34^{+} \mathrm{CD} 1 \mathrm{a}^{-}$human thymic progenitors could develop into ILCs ${ }^{51}$ (Fig. 3c; Supplementary information, Fig. S6a, b).

Our previous study has shown that IL3RA is overrepresented in the presumed thymus seeding progenitors (TSPs) in human fetal liver. ${ }^{46}$ In the present study, we found that IL3RA was also enriched in LPs (LP1 and LP2), suggesting the potential function of IL-3RA on ILC lineage decision (Fig. 3d). By flow cytometric analysis, we found that about one third of immunophenotypically identified lymphoid progenitors $\left(\mathrm{Lin}^{-} \mathrm{CD} 45^{+} \mathrm{CD} 34^{+} \mathrm{CD} 127^{+}\right.$) in fetal liver were IL-3RA ${ }^{+}$. The stronger CD34 expression on IL-3RA ${ }^{+}$lymphoid progenitors indicated the higher hierarchy and more lineage potential of IL-3RA ${ }^{+}$lymphoid progenitors than those of IL-3RA cells (Fig. 3e). We then explored the lineage differentiation potential of the IL-3RA ${ }^{+}$and IL-3RA Iymphoid progenitor cells in fetal liver by co-culture with the OP9 and OP9-DL4 stromal cells (Fig. 3f-k; Supplementary information, Fig. S6c-f). For differentiation assay at bulk level, IL-3RA ${ }^{+}$progenitors generated ten-fold more $\mathrm{CD}_{4} 5^{+}$hematopoietic cells than $\mathrm{IL}-3 \mathrm{RA}{ }^{-}$progenitors coculturing with OP9 or OP9-DL4 stromal cell with IL-7, SCF, FL and IL-15, suggesting the stronger differentiation capacity of IL$3 R A^{+}$cells (Fig. 3f, g; Supplementary information, Fig. S6c, d). Intriguingly, IL-3RA ${ }^{-}$progenitors generated more $\mathrm{CD} 19^{+} \mathrm{B}$ cells than IL-3RA ${ }^{+}$progenitors (Fig. 3f, g; Supplementary information, Fig. S6c, d). Moreover, IL-3RA- progenitors seemed to be largely $B$ cell lineage-committed since majority of the cocultures with IL$3 R A^{-}$progenitors (6 wells of total 9 wells in two independent experiments) generated only B cells (Fig. $3 f, g$ ). However, all the cocultures with IL-3RA ${ }^{+}$progenitors could generate multiple lineage cells, including $\mathrm{CD}_{3} 3^{+}$myeloid cells and $\mathrm{CD} 56^{+} \mathrm{NK}$ cells besides $B$ cells (Fig. $3 f, g$ ). In the OP9-DL4 coculture system, the B lineage differentiation capacity of both lymphoid progenitors was dramatically inhibited by NOTCH signaling, but IL-3RA ${ }^{+}$lymphoid progenitors generate more T cells (Fig. $3 \mathrm{~h}, \mathrm{i}$ ) as well as NK cells and myeloid cells (Fig. 3h, i; Supplementary information, Fig. S6c, d). Collectively, our functional data indicated that IL-3RA ${ }^{+}$progenitors had multiple lineage potentials and IL-3RA- progenitors were mainly B cell committed.

To further confirm the ILC potential of IL-3RA ${ }^{+}$and IL-3RA progenitors, we used lymphoid progenitor cells $\left(\mathrm{Lin}^{-} \mathrm{CD} 45^{+} \mathrm{CD}^{+} \mathrm{CD} 127^{+} \mathrm{CD} 117^{+}\right)$isolated from fetal liver as positive control for ILC-induction coculture experiment. These cells contained ILCP identified in our study (Fig. 3j), and were also phenotypically consistent with the findings from Di Santo's group. ${ }^{36}$ Under the ILCinduction condition (in the presence of IL-2, IL-7, IL-1 $13, \mathrm{IL}-23, \mathrm{IL}-25$ and 


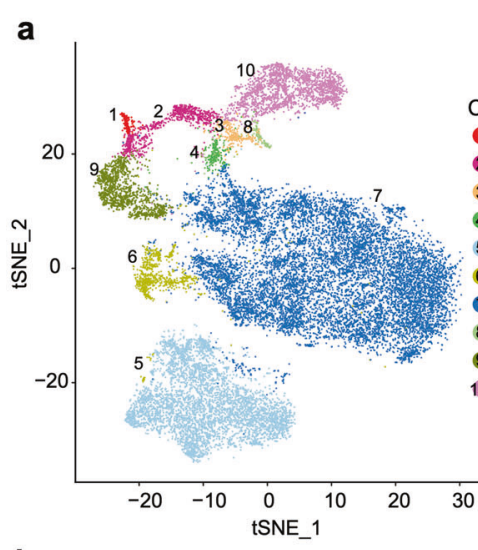

b

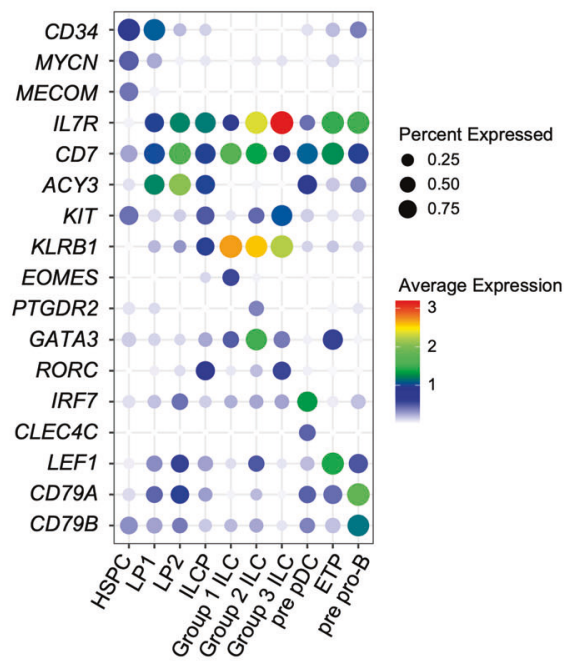

d

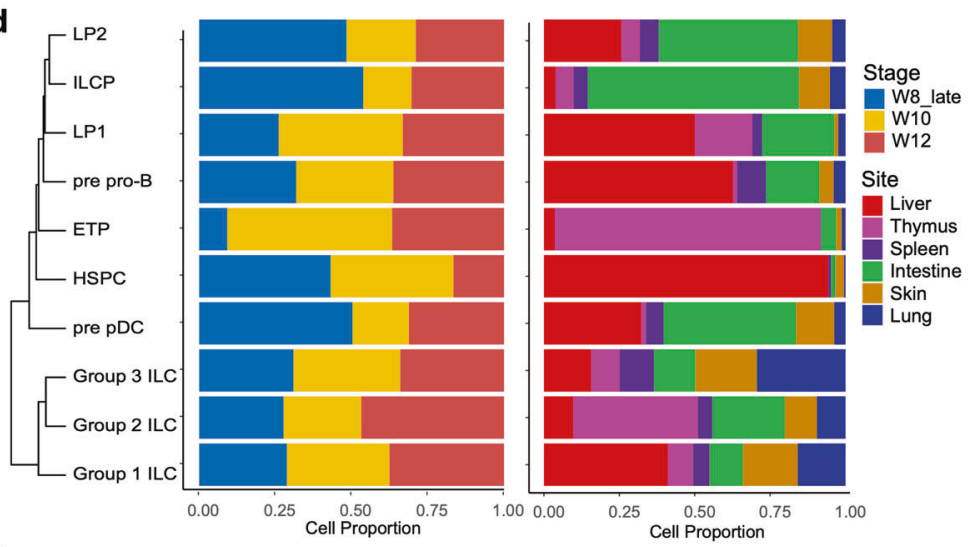

e
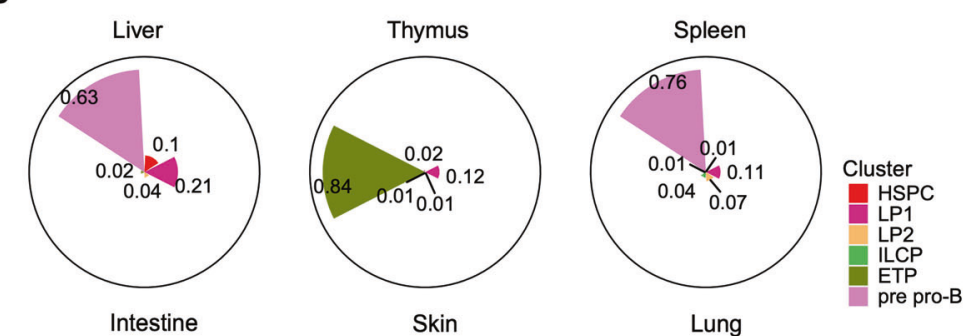
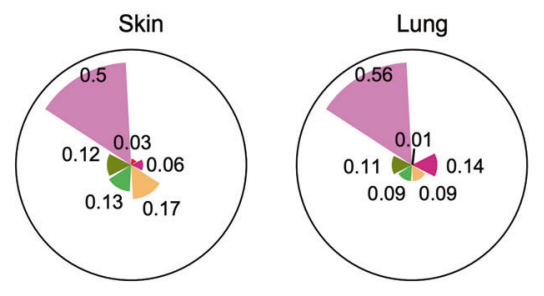

c

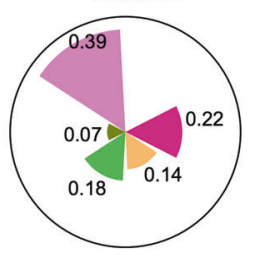

Percent Expressed

25

50 $75 \bigcirc 100$

$\begin{array}{llll}-1 & 0 & 1 & 2\end{array}$
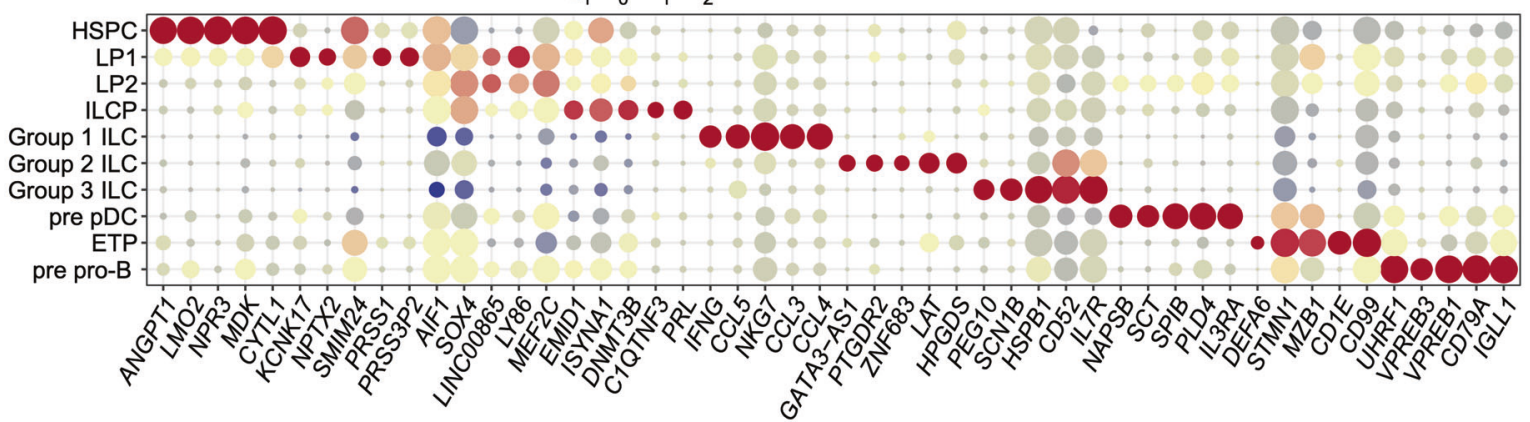

Fig. 2 Single-cell transcriptome profiling of lymphoid-related population in human fetal hematopoietic, lymphoid and non-lymphoid tissues. a $t$-SNE visualization of ten lymphoid-related cell clusters on the scRNA-seq data of cells isolated from human fetal hematopoietic (liver), lymphoid (thymus and spleen) and non-lymphoid (intestine, skin and lung) tissues at 8,10 and 12 PCW. Color indicates the cell identity. b Dot plots show the featured genes expressed in different cell clusters. Colors represent the average gene expression levels, and size encodes the proportion of gene-expressing cells. c Dot plots show the expression level of the top 5 DEGs of each cell cluster. Colors represent the average expression and size encodes the proportion of gene-expressing cells. $\mathbf{d}$ Hierarchical clustering of lymphoid-related cell clusters based on Euclidean distance (left panel). The dynamic change of these populations during embryonic development (middle panel) and their tissue distribution (right panel) are shown in bar graph. Embryonic stages and tissues are indicated by colors. e Pie charts show the proportions of six progenitor clusters in each organ. The radii of sectors indicate proportions of each cell cluster.

IL-33), ILCP generated IFN- $\gamma$-producing ILC1, IL-13-producing ILC2 and IL-17/IL-22-producing ILC3 within $\mathrm{Lin}^{-} \mathrm{CD} 45^{+} \mathrm{CD} 7^{+}$cells consistent with the definition used by Di Santo's group (Fig. 3j). Meanwhile, IFNY-producing ILC1 cells and IL-13-producing ILC2s were generated by IL-3RA ${ }^{+}$lymphoid progenitors, and a few IL-17/IL-22-producing ILC3s were also detected in the IL-3RA ${ }^{+}$cell differentiation products (Fig. $3 \mathrm{j}$, k). More importantly, compared with IL-3RA ${ }^{+}$cells, IL-3RA ${ }^{-}$cells showed much less hematopoietic cell production and IFN- $\gamma$, IL-13, IL$17 \mathrm{a}$ and IL-22-producing cells were hardly observed, which further indicates the lack of helper ILC lineage potential of IL-3RA ${ }^{-}$lymphoid progenitors (Fig. 3j, k).

Single-cell clonal assay showed that IL-3RA ${ }^{+}$lymphoid progenitors represented a heterogeneous population of uni-potent and multipotent progenitors. $7 \%$ of the cultures derived from single IL-3RA ${ }^{+}$progenitors 
a

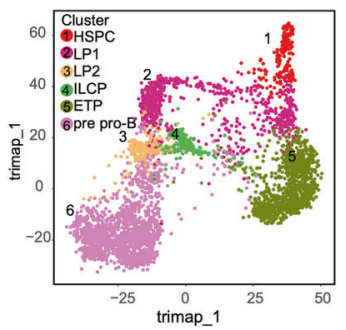

b

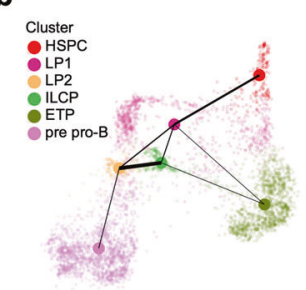

C
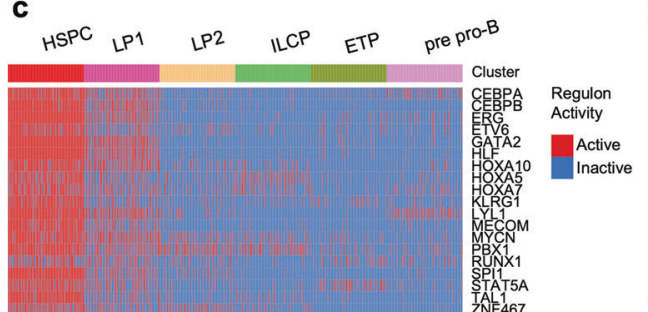

11 PCW Liver Gate from 7-AAD-Lin-CD45+
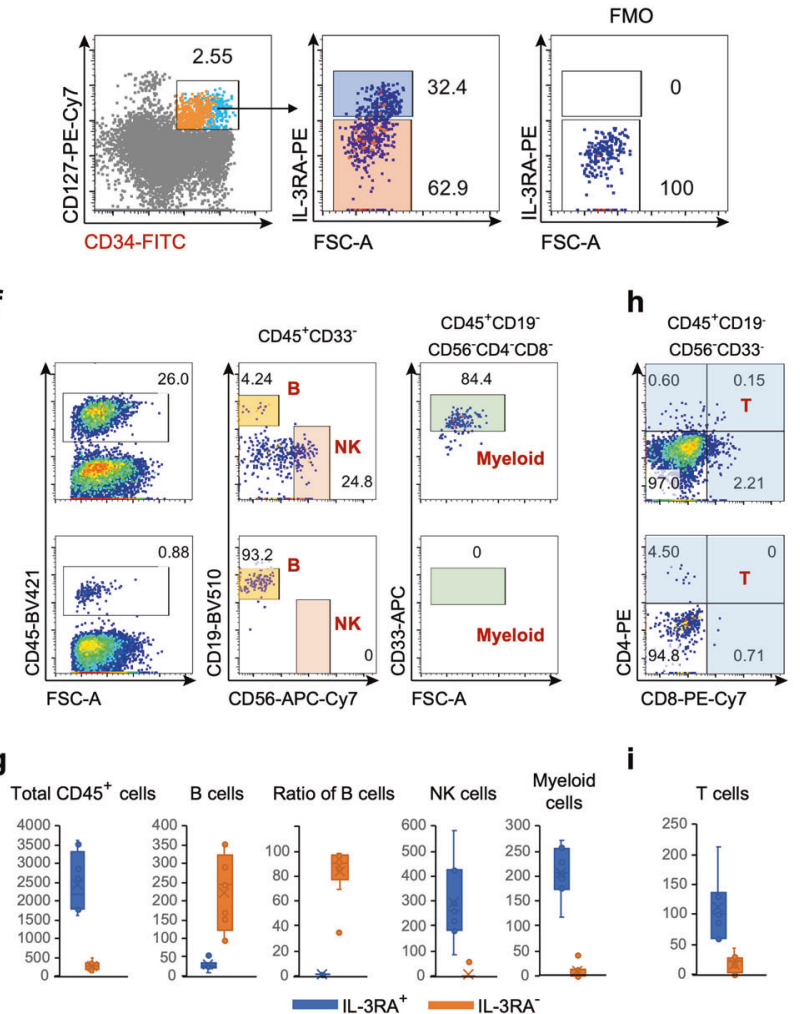

j

Gate from $\operatorname{Lin}^{-} \mathrm{CD} 45^{+} \mathrm{CD} 7^{+}$
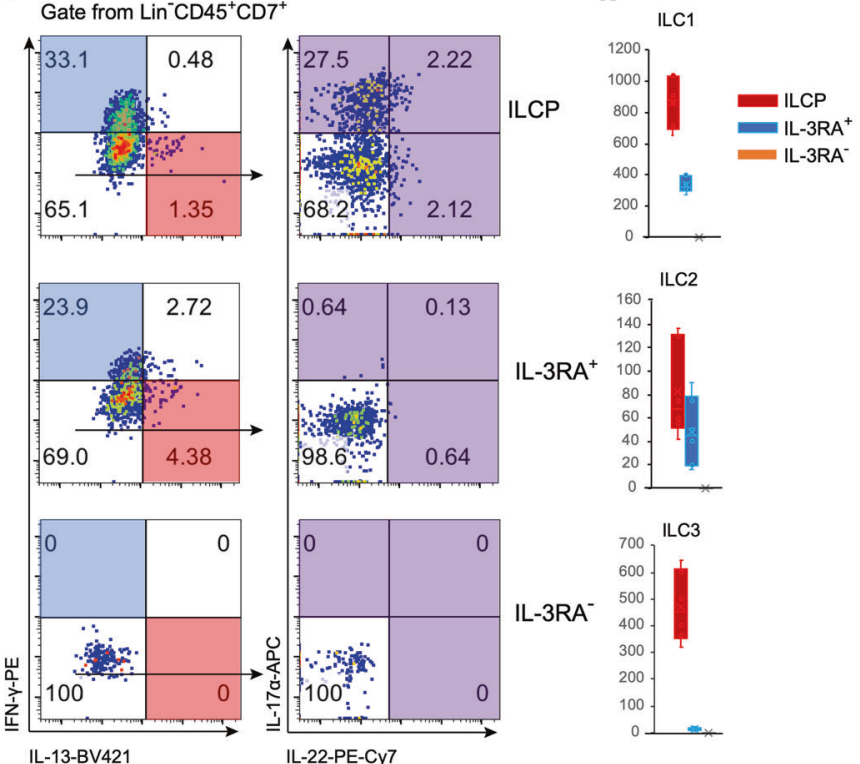

generated only NK cells whereas the remaining cells were multipotent progenitors that could give rise to two or more lineages. Approximately $20 \%$ of the cultures could generate $\mathrm{CD}_{19^{+}} \mathrm{B}$ cells, CD4 ${ }^{+}$and/or $\mathrm{CD} 8^{+}$ $\mathrm{T}$ cells, CD56 ${ }^{+} \mathrm{NK}$ cells and $\mathrm{CD}^{+} 3^{+}$myeloid cells (Supplementary information, Fig. S6e). Under ILC-induction condition, IFN- $\gamma$-producing ILC1 cells, IL-13-producing ILC2s and IL-17a and/or IL-22-producing ILC3s were generated by most of single-cell cocultures with IL-3RA ${ }^{+}$lymphoid progenitor cells. The cell numbers of ILC2 and ILC3 were low, possibly due to the limited ILC induction efficiency of lymphoid progenitors that were not committed to ILC yet. Therefore, the results of ILC-induction at single-cell level were consistent with the functional data at bulk level (Fig. 3j, k; Supplementary information, Fig. S6f).

In summary, our functional data at bulk and single-cell levels indicate the difference of lineage potential between IL-3RA ${ }^{+}$and IL-3RA ${ }^{-} \mathrm{CD} 45^{+} \mathrm{CD} 127^{+} \mathrm{CD} 34^{+}$lymphoid progenitors. IL-3RA ${ }^{+}$lymphoid progenitors showed multi-potent differentiation potentials to $T, B, I L C$ and myeloid lineage, while IL-3RA ${ }^{-}$cells were mainly committed to $B$ lineage. 


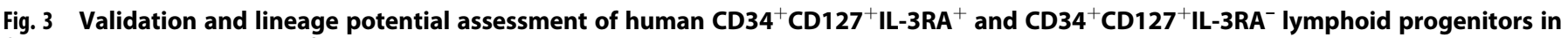
fetal liver. a Visualization of HSPC, LP1, LP2, ILCP, ETP and pre pro-B cell clusters by trimap assay. b Partition-based graph abstraction (PAGA) topology tree of cell clusters. The width of line in PAGA indicates the strength of connectivity between cell clusters. The colors represent cell identities in $\mathbf{a}$ and $\mathbf{b}$. c Heatmap shows the highly expressed TF-encoding genes in progenitor clusters. $\mathbf{d}$ Dot plots show the expression level of top 10 DEGs in HSPC, LP1, LP2, ILCP, ETP and pre pro-B cell cluster in liver. Colors represent the gene expression level and size encodes the proportion of gene-expressing cells. IL3RA highlighted in red is highly expressed in LP1 and LP2 cells. e Flow cytometry plots show stronger CD34 expression in IL-3RA ${ }^{+}$than that in IL-3RA ${ }^{-}$Lin $^{-} \mathrm{CD} 45^{+} \mathrm{CD} 34^{+} \mathrm{CD} 127^{+}$lymphoid progenitors of human fetal liver. Gating strategy for IL3RA was based on Fluorescence Minus One (FMO) control (right panel). f, h IL-3RA ${ }^{+}$and IL-3RA Lin $^{-}$CD45 ${ }^{+}$CD $34^{+}$CD127 $7^{+}$lymphoid progenitors (150 cells for each subset) were sorted and cocultured with SCF, FL, IL-7 and IL-15 on OP9 stromal cells (f) and OP9-DL4 stromal cells (h). The IL-3RA ${ }^{+}$lymphoid progenitors generate more $\mathrm{CD}_{4} 5^{+}$hematopoietic cells and have more NK, myeloid (upper panel in $\mathbf{f}$ ) and $\mathrm{T}$ lineage (upper panel in h) potential while IL-3RA- lymphoid progenitors show $B$ lineage commitment (below panel in $\mathbf{f}$ ) suggested by coculture experiments. B, NK, myeloid and T cells were identified as positive for its respective lineage markers and negative for other lineage markers. g, i Box plots show the numbers of indicated lineage cells, and the percentage of B cells in total CD45 $5^{+}$hematopoietic cells after cocultured for 8 days on OP9 stromal cells $(\mathbf{g})$ or 12 days on OP9-DL4 stromal cells (i). j Fetal liver ILCP $\left(\operatorname{Lin}^{-} \mathrm{CD} 45^{+} \mathrm{CD} 7^{+} \mathrm{CD} 127^{+} \mathrm{CD} 117^{+}, \mathrm{upper}\right.$ panel), IL-3RA ${ }^{+}$(middle panel) and IL-3RA ${ }^{-}$in $^{-} \mathrm{CD} 45^{+} \mathrm{CD} 34^{+} \mathrm{CD} 127^{+}$lymphoid progenitors (below panel) were cultured on OP9-DL4 stromal cells for 10-14 days under ILC-induction condition (IL-2, IL-7, IL-1 $\beta$, IL-23, IL-25 and IL-33). After stimulation with PMA/ionomycin $4 \mathrm{~h}$, cytokine production was analyzed by flow cytometry analysis. IL-17 $\alpha$ and IL-22 producing cells were analyzed after gating on cells negative for IFN- $\gamma$ or IL-13. $\mathbf{k}$ Box plots show numbers of ILC1, ILC2 and ILC3 production of the indicated populations. ILC1, ILC2 and ILC3 were identified as shown in $\mathbf{j}$.

\section{Heterogeneity and tissue distribution bias of Group 1 ILCs}

To delineate the heterogeneity of Group 1 ILC, we further divided these cells into four sub-clusters, from ILC1_a to ILC1_d (Fig. 4a). Group 1 ILC-featured genes, TBX21, NFIL3, EOMES and IFNG were highly expressed in all ILC1 sub-populations, suggestive of their identities (Fig. 4b; Supplementary information, Table S5). Interestingly, our data showed the tissue-preferred distribution of ILC1 sub-clusters, especially of ILC1_b. Cells in the ILC1_b cluster were largely from skin, particularly from the skin of 10 and 12 PCW, probably indicative of the specialized ILC1 state in skin at this stage (Fig. 4c). CXCR4, the gene encoding the receptor of chemokine CXCL12 (SDF-1) expressed on the primary human skin fibroblast, $^{72,73}$ was enriched in ILC1_b cluster that might be involved in the recruitment of ILC1_b to skin (Fig. 4d). Whether CXCL12-CXCR4 interaction also plays a role in ILC1_b development remains elusive. ILC1_c cells were preferably located in liver and spleen (Fig. 4e), with high expression of molecular chaperonecoding genes such as HSPA1B, HSP9OAA1 and HSPA1A, and their function needs further investigation.

Although no distinct NK population was identified in our study due to the universal expression of EOMES in Group 1 ILC cells (Fig. 4b), cytotoxicity-related genes, FGFBP2, FCGR3A (encoding CD16a) and GZMB, were enriched in ILC1_d cluster, highlighting their NK and/or ILC1 identities (Fig. 4d). Further analysis revealed the heterogeneity of ILC1d cells that contained NK and ILC1 subsets, confirmed by curated NK genes scoring (GEO: GSE70580, Fig. 4f). In accordance with our previous study, IL7R was enriched in ILC1 subset, but not in NK cells, and FGFBP2 showed restricted expression in the NK subset (Fig. $4 \mathrm{~g}$ ).

ILCs are considered as the tissue-resident lymphoid cells in mouse and human. At adult stage, the ILC precursors in the secondary lymphoid organs are proposed to give rise to local ILCs, while the developmental event at fetal stage is largely unknown. The cell cycle analysis showed that most of ILC1s were quiescent except for those in ILC1_a (Fig. 4h; Supplementary information, Table S6). The highly proliferative feature of ILC1_a was also evidenced by the high expression of MKI67 and TOP $2 A_{1}^{74}$ the latter gene encoding DNA topoisomerase that regulates the DNA topologic states during DNA replication (Fig. 4d). Given the low expression level of $K L R B 1$, we hypothesized that these cells might be the ILC1 precursors (Fig. 4b). Among all the Group 1 ILC subsets, the proportion of ILC1_a was the highest at week 8 , and then reduced at week 10 and 12, indicating that the development of ILC1_a might occur earlier than other ILC1 subsets (Fig. 4i). Like LP2 and ILCP, ILC1_a cells were enriched in intestine, highlighting the critical role of intestine in ILC development (Fig. 4e). As expected, the predicted putative trajectory started from ILC1_a cells (Supplementary information, Fig. S7a, b), further indicating its precursor identity, followed by three main different development routes toward ILC1_d, ILC1_b and ILC1_b to ILC1_C, respectively. Taken together, our study unveils the heterogeneity of ILC1 and potential ILC1 precursors.

\section{The heterogeneity of conventional and unconventional ILC2 subsets}

The heterogeneity of Group 2 ILC was also investigated. The cluster was separated into five sub-clusters (Fig. 5a; Supplementary information, Table S5), all of which were characterized by the high expression of ILC2-featured TF coding genes, GATA3 and RORA (Fig. 5b). Cells in Pre-ILC2 sub-cluster were proposed to be ILC2 precursors, based on the enrichment of PRSS57 and SPINK2, as well as the reduction of proportion from 8 to 12 PCW (Fig. $5 \mathrm{c}$; Supplementary information, Fig. S8a). Pre-ILC2 were further subgrouped into Pre-ILC2a and Pre-ILC2b, both of which expressed SPINK2 and PRSS57 while proliferating-related gene MKI67 and ILC2-specialized genes PTGDR2 and BCL11B were enriched in the latter one (Supplementary information, Fig. S8b, c), indicating that Pre_ILC2b might be derived from Pre_ILC2a. It was conceivable that Pre_ILC2a has multipotent capability, which needs to be further deciphered by functional experiments. Two conventional ILC2 sub-clusters, CRTH2_ILC2 and PTGS2_ILC2, were identified. Both of them expressed high level of PTGDR2 (encoding CRTH2), while the latter specifically overrepresented PTGS2 that is essential for human ILC2 activation in PB and tonsil (Fig. 5b, c). ${ }^{75}$ Highest expression of ILIRL1 (encoding IL-33 receptor) and ILI3 in CRTH2_ILC2 (Fig. 5C, e) indicated their mature status. Enrichment of NR4A2 and NR4A3 suggested that PTGS2_ILC2 might be transcriptionally correlated with the newly identified ILC2 subpopulation localized with the fibroblast-like adventitial stromal cells $^{76}$ (Fig. 5e). Its skin-resident characteristics together with expression of KIT are consistent with the previous flow cytometric analysis (Fig. 5c, d; Supplementary information, Fig. S8d). Two unconventional ILC2 subsets with low PTGDR2 expression (KIT_ILC2 and CCR9_ILC2), were also identified (Fig. 5a-c). Considering the recently reported ${\mathrm{c}-\mathrm{Kit}^{\mathrm{hi}} \mathrm{CCR6}}^{+}$ ILC2 subset which can convert into IL-17-producing NKp44 ILC3-like cells, ${ }^{77,78}$ we hypothesized that KIT_ILC2 cells which also expressed high level of CCR6 and $L T B$, might be the fetal counterparts of the $\mathrm{c}-\mathrm{Kit}^{\mathrm{hi}} \mathrm{CCR}^{+}{ }^{+}$ILC2 cells with ILC2-ILC3 plasticity.

CCR9_ILC2 was featured by the enrichment of BCL11B and CCR9 (Fig. 5b, c). BCL11B is the critical TF for both T cell commitment ${ }^{79}$ and maintenance of the genetic and functional programs of ILC2, $26,80,81$ and CCR9 is essential for intrathymic seeding of T progenitors. ${ }^{82-84}$ Intriguingly, majority of these cells were from thymus, which might be 

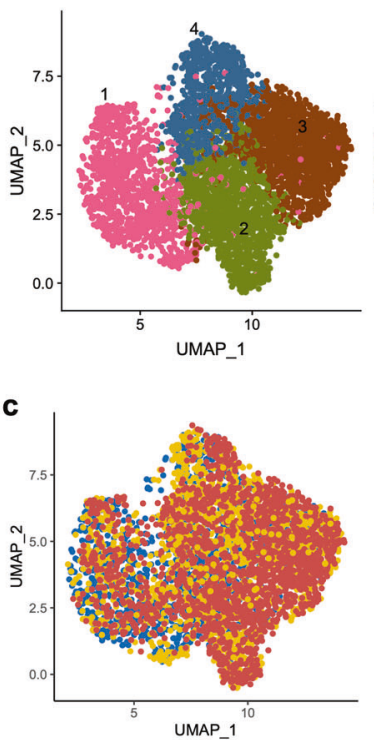

e
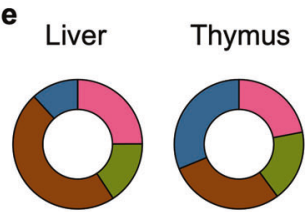

Intestine

Skin
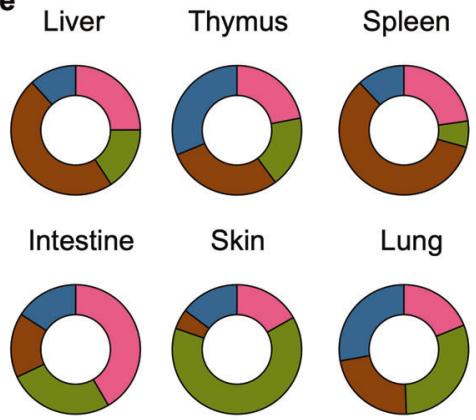

Lung
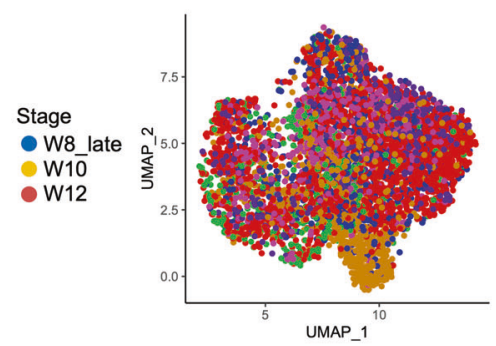

Site

- Liver

- Thymus

- Spleen

Intestin

Skin

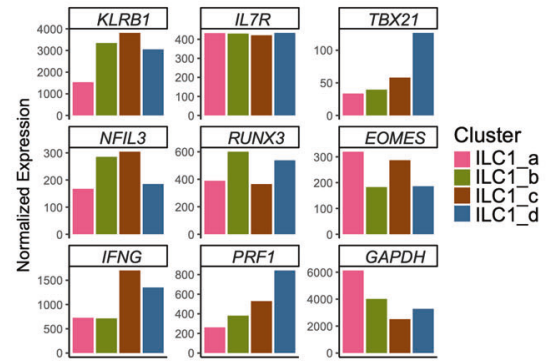

f
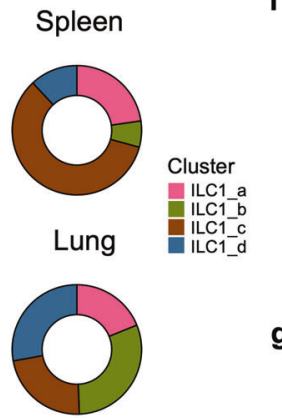

h

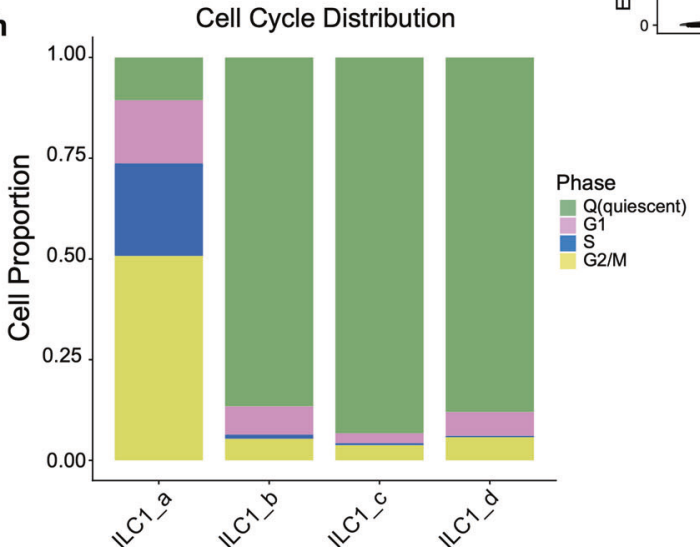

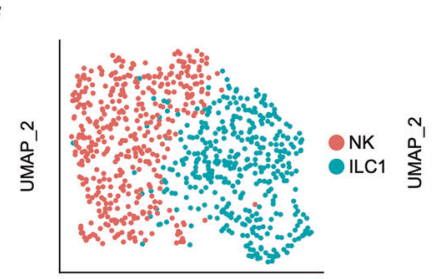

UMAP_1

g

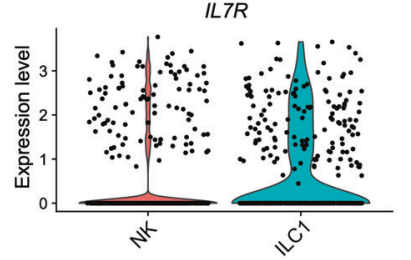

d

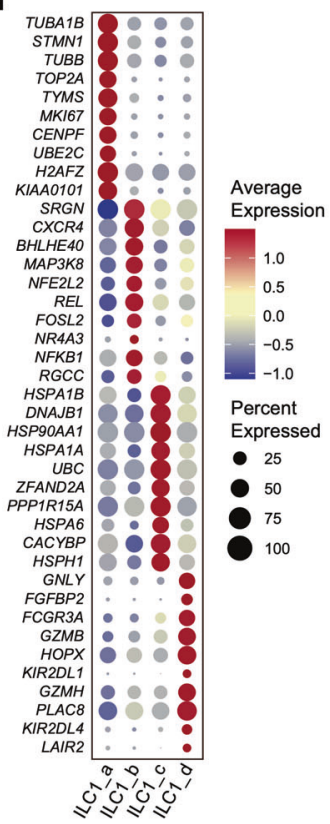

NK_score

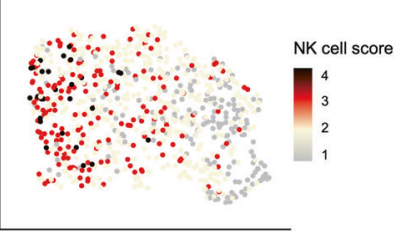

UMAP_1

FGFBP2
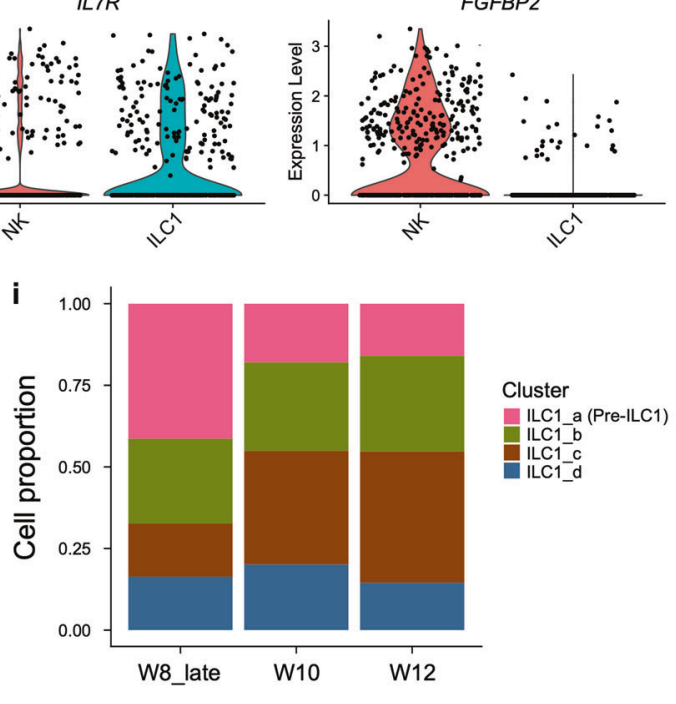

Cluster

ILC1_a (Pre-ILC1)

ILC1_b

ILC1_C
ILC1_d

Fig. 4 Heterogeneity and tissue distribution bias of Group 1 ILCs. a UMAP visualization of Group 1 ILCs from Fig. 2a with re-clustered

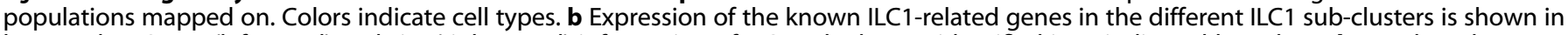
bar graph. c Stage (left panel) and site (right panel) information of ILC1 sub-clusters identified in a, indicated by colors. $\mathbf{d}$ Dot plots show top

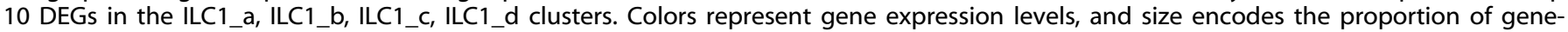
expressing cells. e Pie charts show the proportions of ILC1_a, ILC1_b, ILC1_c and ILC1 d in tissues. Colors represent cell clusters described in a. f UMAP visualization of the cells in ILC1_d cluster are further separated into NK and ILC1 subgroup by UMAP analysis (left panel). NK score (GEO: GSE70580) is projected onto UMAP visualization, and colors indicate the expression level of NK-related genes (right panel). $\mathbf{g}$ Violin plots show the expression level of IL7R and FGFBP2 in NK and ILC1 subgroups defined in $\mathbf{f}$. $\mathbf{h}$ The proportions of cell in different cell cycle statues in

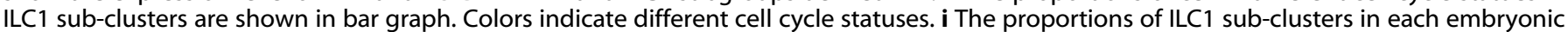
development stages are shown in bar graph. Colors indicate cell types. 
1114

due to the CCR9-CCL25 interaction. In a previous study, we have identified a unique ILC population (ILC-C2) in human fetal thymus (GEO: GSE133341), ${ }^{46}$ showing the high expression of T cell-featured genes (CD3G and CD4), and ILC2-related genes (GATA3 and IL4). Expression of CD1E, CD2 and CD8A in CCR9_ILC2 also suggested its close relationship with T cells (Fig. 5e). After being projected to UMAP with all Group 2 ILC cells from the present study, these ILC-C2 cells were clustered with CCR9_ILC2, further validating their ILC2 identity (Fig. 5f). To further examine whether this unique CCR9_ILC2 subpopulation could be captured at protein level, we performed flow cytometric analysis and found that among the

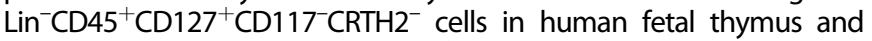
intestine, there was a minor $\mathrm{CCR}^{+}$population in both tissues that was phenotypically consistent with CCR9_ILC2 identified by the transcriptome data, with relatively higher proportion in thymus (Fig. $5 \mathrm{~g}$ ). Next, we exanimated the expression of ILC-specific TFs of this population by flow cytometry. Similar to the conventional ILC2s $\left(\mathrm{Lin}^{-} \mathrm{CD} 45^{+} \mathrm{CD} 127^{+} \mathrm{CD} 117^{-} \mathrm{CRTH} 2^{+}\right), \mathrm{CCR}^{+}$ILC2 had higher expression of GATA3 but lower expression of RORyt than ILC3 cells $\left(\mathrm{Lin}^{-} \mathrm{CD} 45^{+} \mathrm{CD} 127^{+} \mathrm{CD} 117^{+} \mathrm{CRTH} 2^{-}\right.$) (Fig. 5h). Taken together, using transcriptomic and immunophenotypic analysis, we find a new CCR9 ${ }^{+}$PTGDR2 ${ }^{-}$ILC2 population at fetal stage.

\section{Computational prediction of LTi-like cell and Non-LTi ILC3 pathways from proliferating ILC3 precursors}

In accordance with flow cytometric results, Group 3 ILC was the major ILC population with five subpopulations (Fig. 6a; Supplementary information, Table S5), although the expression of NCR2 (encoding NKp44) in ILC3 cells was very low, consistent with the protein expression pattern (Supplementary information, Figs. S2c and S9a). ILC3 a was a proliferating subset, showing the higher expression of DNA replication-regulated genes (TUBA1B, TUBB and TOP2A) and $M K I 67$, but lower expression of ILC3-related genes, such as RORC and $K I T$, than other subsets (Fig. $6 \mathrm{~b}, \mathrm{c}$; Supplementary information, Fig. S9a), indicative of its precursor identity. Meanwhile, the dynamic change of ILC3_a proportion was similar to ILC1 precursor (Figs. 4i and $6 e)$. On the other hand, the proportion of ILC3_e gradually increased along with development, indicating that this subset might represent the mature ILC3s, and higher expression of KLRB1 in ILC3_e also suggested that they might be fully mature (Fig. $6 \mathrm{~b}-\mathrm{e}$ ). Intriguingly, ILC3_e also highly expressed genes encoding NK and T cell surface markers and TFs, such as NKG7, CD 3D, IKZF $3^{85}$ and $B C L 11 B$, as seen in CCR9_ILC2s identified above (Fig. 6d). ILC3_b was featured by KRT81 and CCL5. The LTi-featured genes LTB was expressed in both ILC3_b and ILC3_c cells (Fig. 6d).

To determine the correlation of these five ILC3 subsets, pearson correlation analysis was employed. The results showed two modules, one gathering ILC3_b and ILC3_c, and the other with ILC3_d and ILC3_e. ILC3_e showed the least similarity with ILC3_a compared with the other ILC3 subsets (Fig. 6f). This result promoted us to propose the divergent developmental pathways of LTi and non-LTi ILC3 as known in mouse. Group 3 ILC cells were re-grouped into three major subclusters, named Pre-ILC3, LTi and Non-LTi ILC3-related clusters, by components analysis (PCA) (Fig. 6g). Consistent with pearson correlation analysis results, Pre-ILC3 approximately overlaid with ILC3_a. ILC3_b and ILC3_c formed the LTi-related cluster, with increased expression of LTi characteristic genes such as LTA and LTB. $N R P 1$, expressed by LTi-like group 3 ILCs in smokers' lungs and associated with ectopic lymphoid aggregates, ${ }^{86}$ was also enriched in ILC3_b and ILC3_c (Fig. 6h). Importantly, ILC3_b and ILC3_c were preferably located in lymphoid tissues including thymus and spleen, as well as intestine where $\mathrm{mLNs}$ will be formed in the second trimester (Fig. 6i; Supplementary information, Fig. S9b), correlating the function of $\mathrm{LTi}$ to induce the formation of lymphoid tissues. As expected, ILC3_d and ILC3_e clustered together, forming the Non-LTirelated ILC3 developmental pathway, with expression of CD96 and ITGAE, ${ }^{11,87,88}$ the genes to promote NKp44 ${ }^{+}$ILC3 adhesion to epithelial cells. Chemokine receptor-encoding gene CCR6, which has

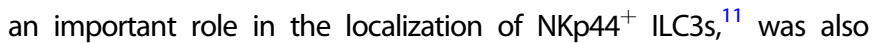
highly enriched in both ILC3_d and ILC3_e (Fig. 6h). In summary, our data illuminate the branched development pathways of LTi and nonLTi ILC3s from ILC3 precursors in early human early fetal stages, which is consistent with mouse studies. ${ }^{89,90}$

\section{TF network and dynamics of cell cycle state along with ILC development}

The annotation of ILCP in our study was based on the reported features of human ILC progenitors/precursors in two previous studies. The ILC progenitors identified in human tonsil with $\mathrm{CD} 34^{+} \mathrm{CD} 117^{+} \mathrm{IL}-$ $1 \mathrm{R} 1^{+}$phenotype also expressed high level fo RORC. Meanwhile, ILCPs are identified as a subset of $\mathrm{Lin}^{-} \mathrm{CD} 45^{+} \mathrm{CD} 7^{+} \mathrm{CD} 127^{+} \mathrm{CD} 117^{+}$cells in human peripheral blood, adult and fetal tissues (Supplementary information, Fig. S10a). ${ }^{21,36}$ Three types of ILC lineage specific precursors were also annotated, predicted by their distinctive proliferating status together with low expression of respective ILC featured genes. To uncover the molecular basis operating ILC lineage commitment, we combined these ILC precursors with ILCP for further analysis. These four cell populations formed distinguished clusters based on their original identities, visualized by $t$-SNE (Fig. 7a; Supplementary information, Fig. S10b and Table S7). In coincidence with studies in mouse, ${ }^{25,27}$ ILCP had the upregulated expression of NFIL3, ID2 and TOX (Fig. 7b), indicating that the multi-potent lymphoid progenitors had been specialized to the ILC lineages. The expression of ILC lineage-specific TF-coding genes, including EOMES, GATA3, and $A H R$, remained low at ILCP stage, and was subsequently upregulated in the Pre-ILC1, Pre-ILC2 and Pre-ILC3 cells, respectively (Fig. 7b). Notably, TCF7, encoding the important TF (TCF1) for $T$ cell specialization both in human and mouse, ${ }^{46,91}$ was also highly expressed in murine early innate lymphoid progenitors (EILPs) with restricted ILC potential to generate both NK and helper ILCs. ${ }^{19,92}$ In addition, in $\mathrm{Tcf}^{\prime-}$ mice, Ly49a ${ }^{+} \mathrm{NK}$ cells, ILC2s and immature ILC2s, were sharply reduced and ILC2-mediated type 2 immune responses to helminth infection was lacking ${ }^{93-95}$ highlighting the importance of TCF7 on ILC2 development and function. However, TCF7 was barely expressed in ILCP but highly expressed in Pre-ILCs, especially Pre-ILC3 (Fig. 7b), suggesting that at human early fetal stages, TCF7 might be involved in distinct helper ILC lineage restriction, but not essential for common ILC lineage commitment or development of killer ILCs. Expression of ZBTB16 (encoding PLZF) discriminates ILC precursors without LTi potential from ID2 highly expressing common helper ILC progenitors (CHILP) in murine. ${ }^{29,33}$ ZBTB16 expression level was low in ILCP but high in Pre-ILC2 and Pre-ILC3; meanwhile, ZBTB16 expression was also low in Pre-ILC1 that could generate NK cells (Fig. 7b), indicating that ZBTB16 may play an important role in LTi development at human early fetal stages. Additionally, IRF8, encoding the essential TF for DC development and reported to be expressed in subsets of $\mathrm{ETPs}^{46,59,69}$ was also enriched in ILCP, suggesting the strong correlations among the development pathways of ILCS, T cells and DCs (Fig. 7c). Many T cell-related TFs were expressed in both ILCP and ILC precursors, including BCL11A and TCF3 in ILCP, LEF1, ETS1 and IKZF3 in Pre-ILC1, LYL1 and BCL11B in Pre-ILC2 and TCF7 in PreILC3 ${ }^{61,96,97}$ (Fig. 7c; Supplementary information, Fig. S10c), suggesting the involvement of similar transcription machinery for T cell and ILC development. $^{98}$

Consistent with their annotations, ILCP showed less primed lineage potential than the three individual ILC precursors that were restricted to the respective ILC lineage branch, predicted by STEMNET. Notably, Pre-ILC1 cells had both ILC1 and NK cell potentials (Fig. 7d, e). Interestingly, cell cycle analysis showed that the majority of ILCPs were quiescent (Fig. 7f; Supplementary information, Table S6). In contrast, most of ILC precursors were in active cell cycle status, as mentioned above. The proportion of resting cells varied among three types of ILC precursors, and that in Pre-ILC1 and Pre-ILC3 was similar, while Pre-ILC2 showed the highest proportion. Pearson correlation analysis showed that ILCP was less similar to Pre-ILC1, which might be due to the NK precursors in ILC1_a cells (Fig. 7g). These data were 
a

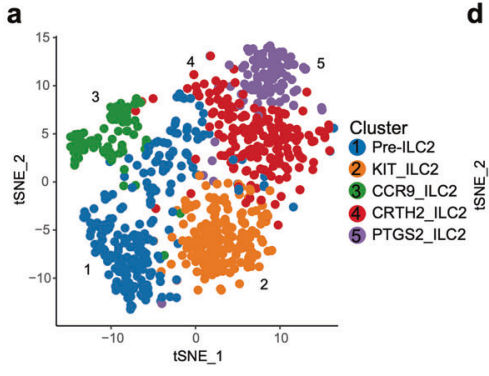

b
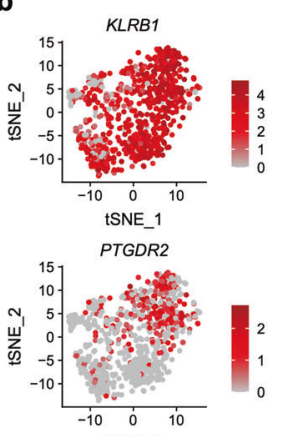

tSNE_1

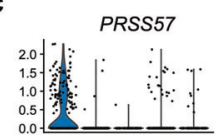

KIT
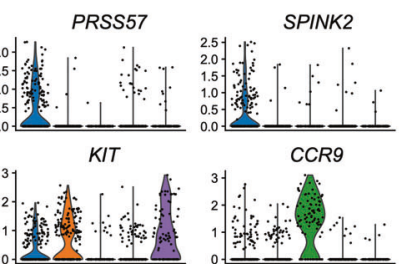

CCR9
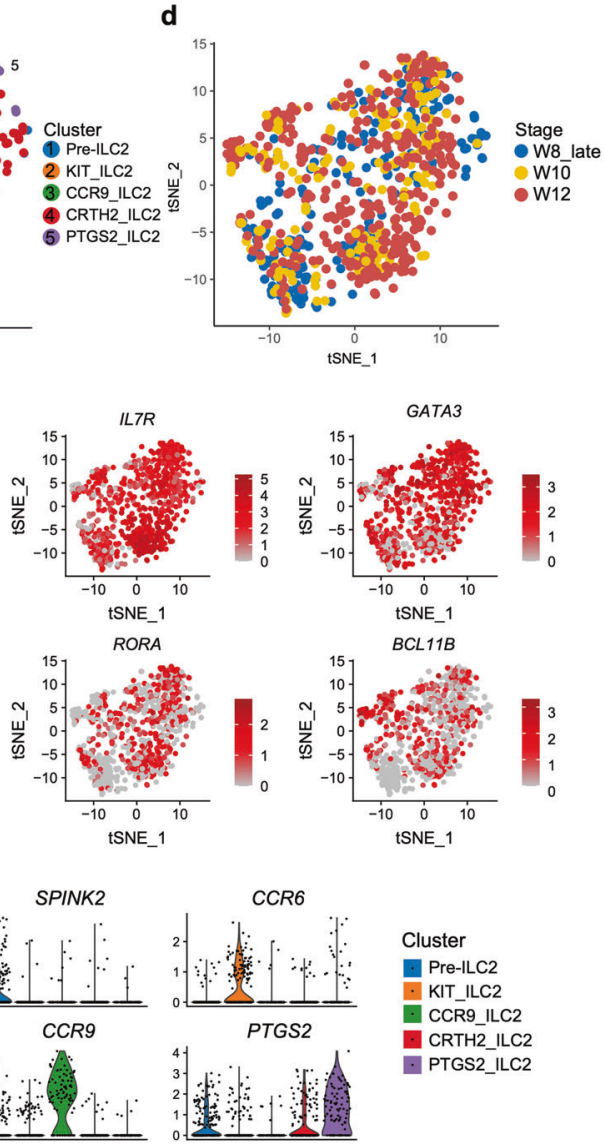
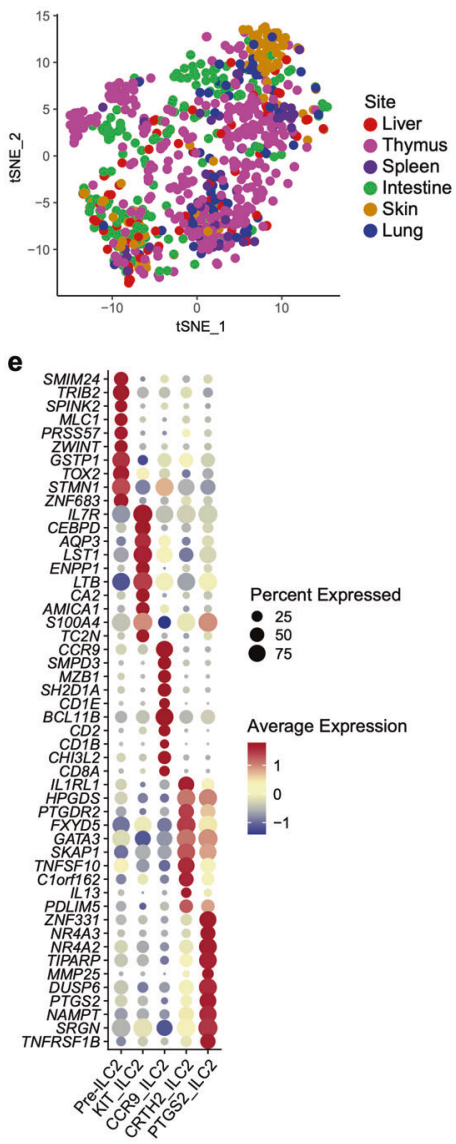

g

f
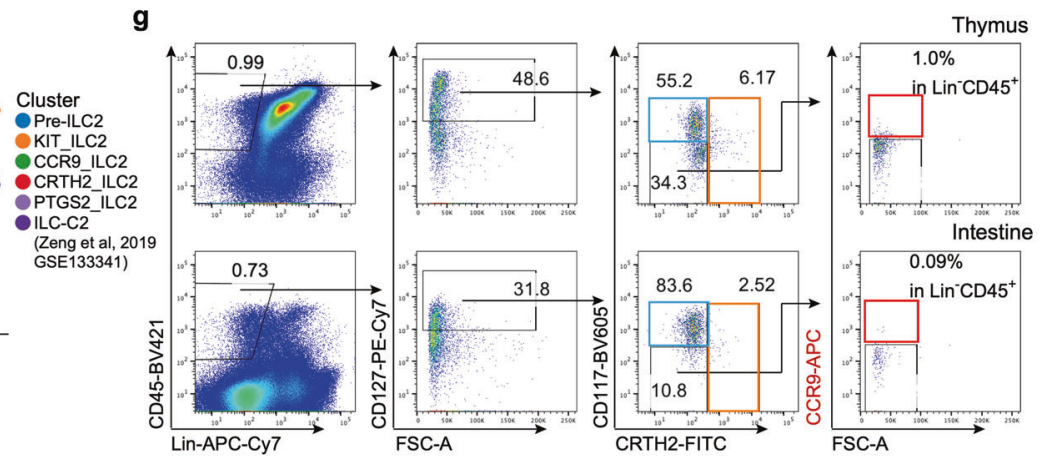

h
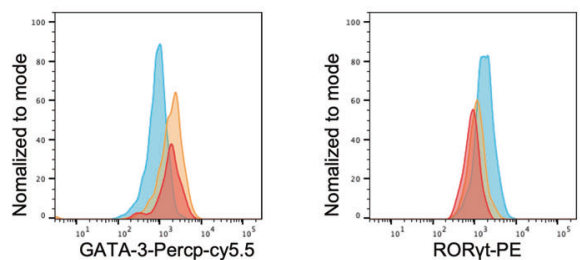

KitCRTH2-CCR9 KitCRTH2 $2^{+}$ILC2
Kit+CRTH2 ILC3

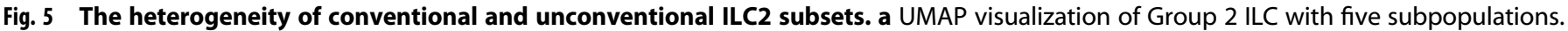

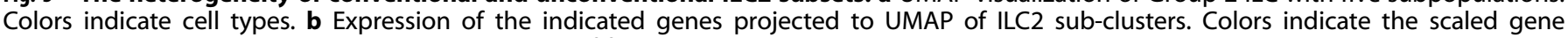
expression level. c Violin plots show the expression of featured genes in ILC2 sub-clusters. Colors represent cell clusters. d UMAP visualization

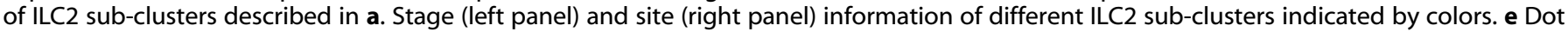

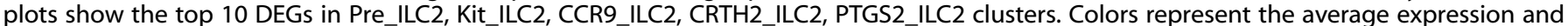
size encodes the proportion of gene-expressing cells. f ILC-C2 cluster in human fetal thymus identified previously (GEO: GSE133341) is projected to UMAP of ILC2 sub-clusters. ILC-C2 cells are highlighted and the ILC2 cells in this study are in gray (below panel). $\mathbf{g}$ Representative flow cytometry results show the frequencies of CCR9_ILC2 in fetal thymus (upper) and intestine (below). CCR9_ILC2 cell is gated as

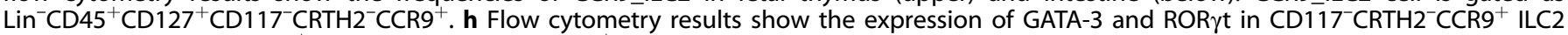
(red), conventional CRTH2 ${ }^{+}$ILC2 (orange) and $\mathrm{CD}_{11} 17^{+} \mathrm{CRTH} 2^{-}$ILC3 (blue) cells. 


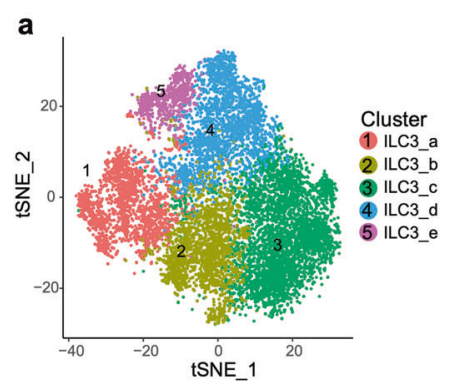

b
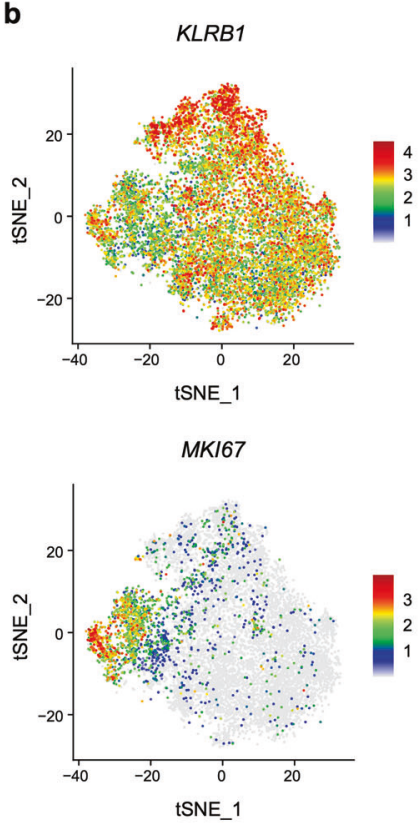

g

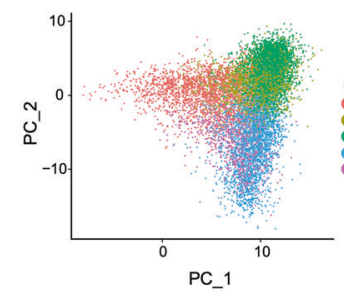

c

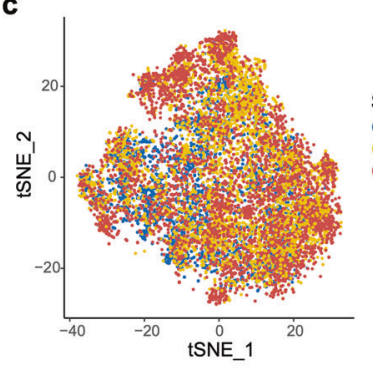

d

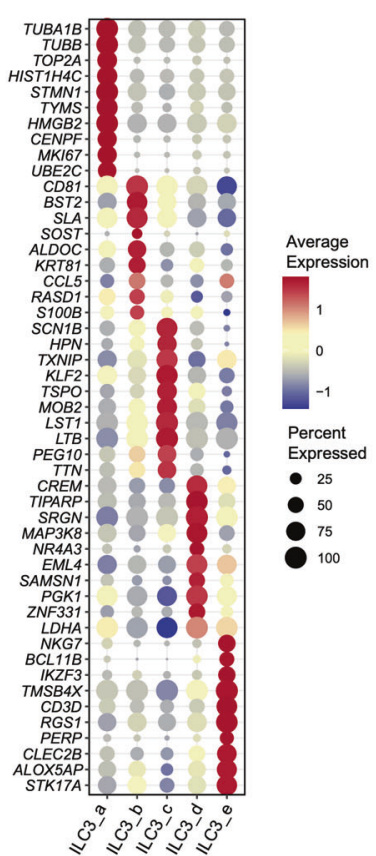

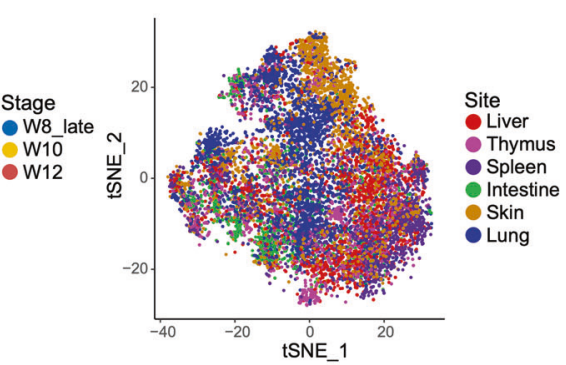

e

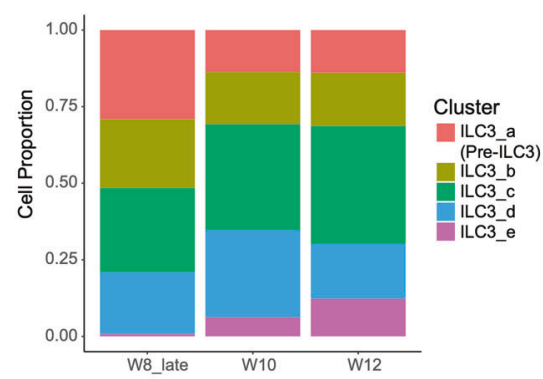

f

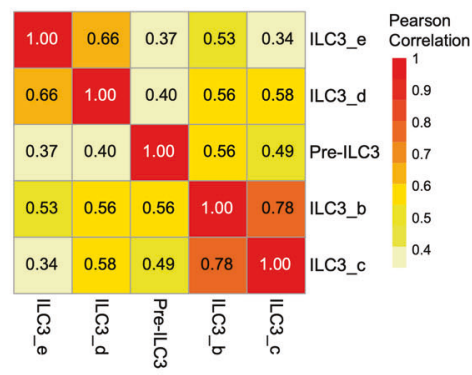

h

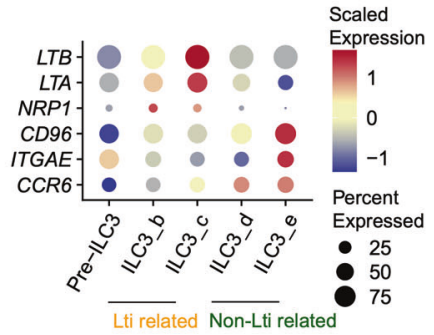

i

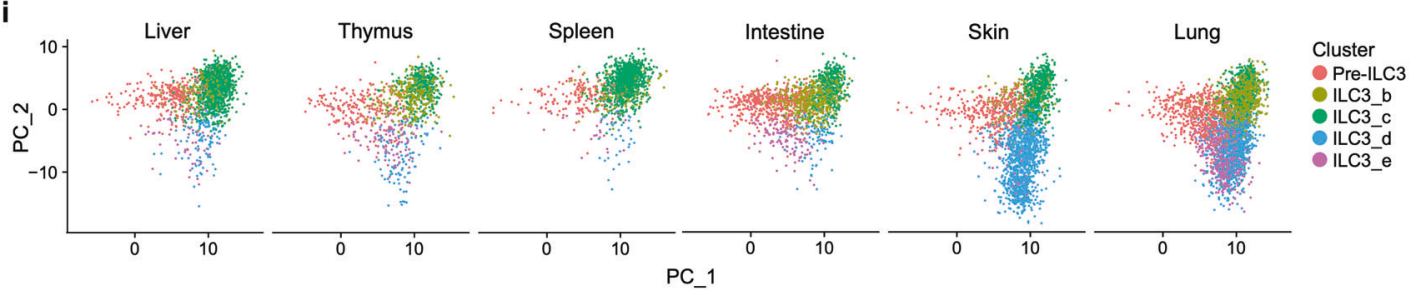

Fig. 6 Data analysis reveals LTi-like and non-LTi development pathways from proliferating Pre-ILC3. a $t$-SNE visualization of Group 3 ILCs with re-clustered subpopulations. Colors indicate cell subpopulations. b Expression of KLRB1 and MKI67 in ILC3 cells projected onto $t$-SNE. Colors indicate gene expression levels. c $t$-SNE visualization of ILC3 sub-clusters described in a, with stage (left panel) and site (right panel) information indicated by colors. d Dot plots show the expression level of top 10 DEGs in ILC3_a, ILC3_b, ILC3_c, ILC3_d, ILC3_e. Colors represent the average expression and size encodes the proportion of gene-expressing cells. e Bar graph shows the proportions of ILC3_a (PreILC3), ILC3_b, ILC3_C, ILC3_d, ILC3_e in different development stages. Colors indicate different cell types. f Heatmap of pearson correlation matrix among five ILC3 sub-clusters. Colors and numbers indicate correlation coefficient. g PCA visualization of all ILC3 sub-clusters (left panel), which can be further clustered into Pre-ILC3, LTi-related and non-LTi groups based on PCA analysis (right panel). $\mathbf{h}$ Dot plots show the scaled expression level of indicated genes in ILC3 sub-clusters. Colors represent the scaled expression and size encodes the proportion of gene-expressing cells. $\mathbf{i}$ The tissue distributions of ILC3 sub-cluster were projected to PCA. Colors indicate different ILC3 sub-clusters. 
a

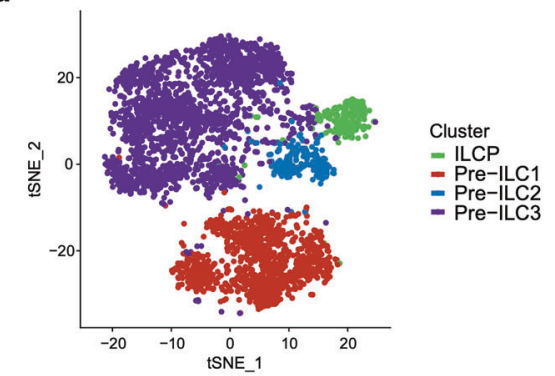

b

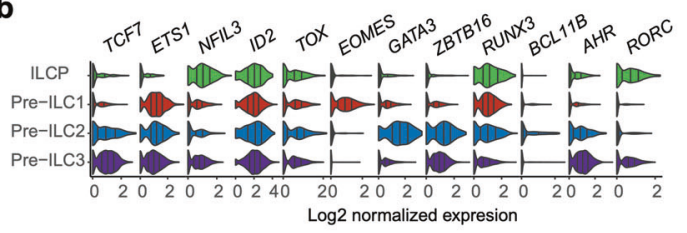

d
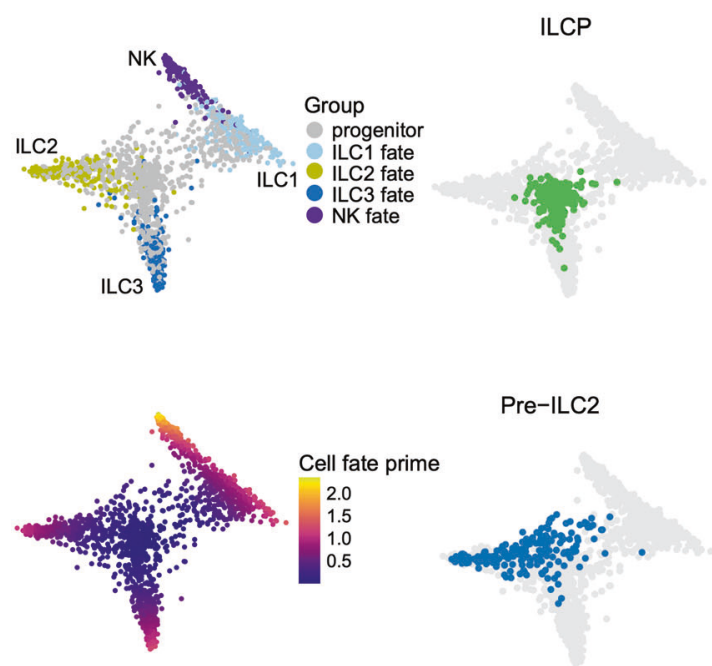

C

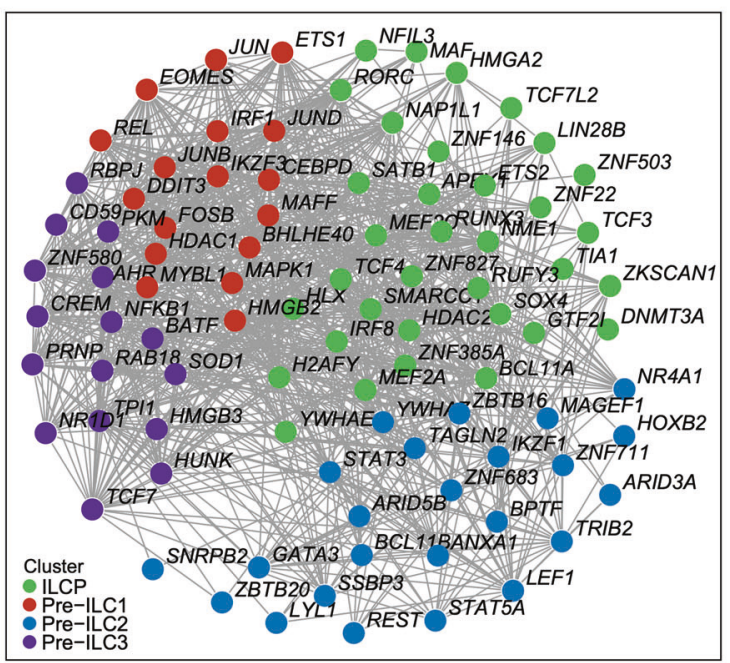

f

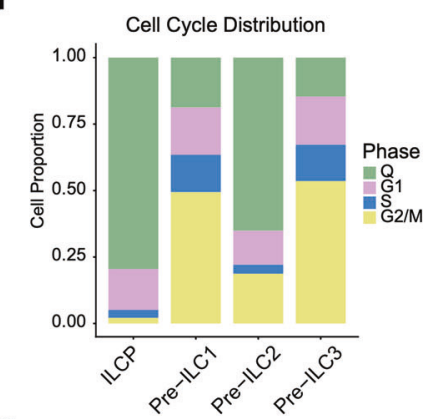

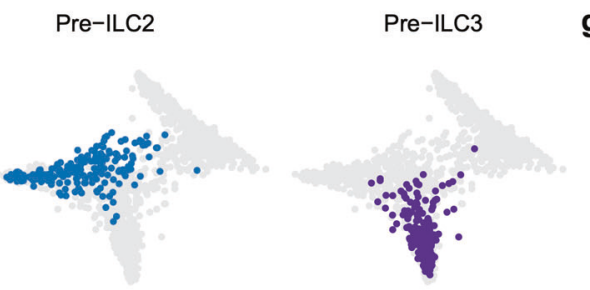

Fig. 7 TF network and dynamics of cell cycle state along with ILC specialization. a $t$-SNE visualization of ILCP from Fig. $2 \mathrm{a}$, Pre-ILC1 from Fig. 4a, Pre-ILC2 from Fig. 5a and Pre-ILC3 from Fig. 6a. b Violin plots show the expression of featured TF-encoding genes in different cell clusters. c The correlation network of TFs of ILCP and three Pre-ILCs. All TFs in each cluster were used to construct network; nodes (TFs) with more than 3 edges are shown. Colors indicate cell types (a-c). d Fully annotated STEMNET map visualizes fate probabilities of progenitors toward mature NK, ILC1, ILC2 and ILC3 cells. Colors indicate cell types (upper panel) and the possibility of progenitor cells developing into the corresponding lineage (below panel). e Visualization of the probabilities of different progenitors and precursors to develop toward the specific lineages. Colors indicate cell types. $\mathbf{f}$ Proportions of the cells with different cell cycle statuses in each cluster are shown in bar graph. Colors represent cell cycle statues. g Heatmap of pearson correlation matrix among ILCP and three Pre-ILCs. Colors indicate correlation coefficient.

supported by the early bifurcation of developmental pathways of cytotoxic ILCs and helper ILCs, which is conserved in mouse and human. In summary, we characterize the TFs that might govern the ILC lineage decision at ILCP to ILC precursor stage, accompanied by dynamic cell cycle status.

\section{DISCUSSION}

ILCs, the innate counterparts of T cells, are increasingly appreciated due to their irredundant role in host immune system. The developmental pathways of ILCs in mouse have been explored in recent years, however, the ontogeny of human ILCS is poorly understood. In this study, we investigated human ILC development at fetal stage by scRNA-seq and biological validation. A linear and successive developmental path from HSCs, to LP1, LP2, ILCP, and to each ILC-specific precursor was revealed by our data. Due to the limited samples, the precise progenitor-progeny relationship of these progenitor/precursors needs further investigation.

HSCs go through the sequential restriction of multi-lineage differentiation potential, resulting in lineage commitment during hematopoiesis, the process controlled by multiple-layer of regulatory mechanisms. In adult human bone marrow and cord blood, the lymphoid-primed progenitors still retain myeloid potential but not erythroid potential. ${ }^{99-101}$ These progenitors, named "lymphoid-primed multipotent progenitors" or "multilymphoid progenitors" (MLP), were identified as high expression 
1118

of L-selection or $\mathrm{CD}^{2} 4^{+} \mathrm{CD} 38^{-}{ }^{-}$Thy-1 ${ }^{-} \mathrm{CD} 45 \mathrm{RA}{ }^{+} .{ }^{100,101}$ The mouse LMPPs are featured by CD135 expression compared with HSCs. ${ }^{102,103}$ Up-regulation of CD127 leads to the final commitment to lymphoid lineage and elimination of myeloid potential. T, B and ILC cells lineage fate might be decided thereafter. $\mathrm{CD}_{135^{+}} \mathrm{a} 4 \mathrm{B7}^{-} \mathrm{HSA} \mathrm{A}^{\mathrm{int}}$ unbiased CLPs, the direct progeny of LMPPs, give rise to $\mathrm{HSA}^{\text {hi }}$ and HSA ${ }^{\text {lo }}$ CLPs which are B-biased and T/ILC biased, respectively. ${ }^{104}$ ILC lineage commitment is further concreted, and the up-regulation of a4 $\beta 7$ and loss of CD135 expression suggest the loss of $B$ and $T$ cell potential successively. ${ }^{105} \mathrm{CD} 127^{+} \mathrm{CD} 117^{+}$ILCPs discovered by Di Santo's group have been proved to be HSC-derived through transplantation experiments, ${ }^{36}$ but the definitive intermediate stage that bridges HSCs and ILCPs was lacking. IL-3RA ${ }^{+} \mathrm{CD} 127^{+} \mathrm{CD} 34^{+}$lymphoid progenitors identified at transcription and protein levels in this study might be the fetal human equivalents of LMPPs mentioned above, regarding their tri-lymphoid and myeloid potential predicted by STEMNET analysis as well as functional validation at bulk and single-cell levels. The IL-3RA ${ }^{+}$lymphoid progenitors might be correlated with the first wave thymus-seeding progenitors with bipotent T/ILC potential as reported in the mouse. ${ }^{106,107}$ Diminished DC potential along ILC lineage commitment has been found in human tonsil and mouse bone marrow. ${ }^{19,21}$ Based on the increased expression of IRF7 and IRF8 together with the decreased expression of IL7R and CD7 compared with LP1, the LP2 cluster might represent the intermediate stage between multi-potent lymphoid progenitors and ILC-committed progenitors. These cells might be equivalent to the specified early innate lymphoid progenitors (EILPs), possessing both ILC and DC potentials, named by Bhandoola's group. ${ }^{19}$ However, the difference of lineage restrictions between LP1 and LP2 needs to be further investigated.

Single-cell clonal assay in the previous study using fetal liver $\mathrm{Lin}^{-} \mathrm{CD} 45^{+} \mathrm{CD}^{+} \mathrm{CD} 127^{+} \mathrm{CD} 117^{+}$cells $^{36}$ which contained ILCP and Pre-ILC3 identified in this study, showed their heterogeneous ILC differentiation potential in vitro. $56 \%$ and $39 \%$ clones from OP9 and OP9-DL4 coculture systems respectively, showed ILC3 uni-product, suggesting that the presence of ILCPs that have already committed to ILC3 lineage. Less than $10 \%$ of these cells had multi-lineage potential to generate all groups of ILCs suggested by both coculture systems. Potential markers to distinguish each ILC precursors have been provided, however, the ideal surface markers to perfectly differ PreILC3 from ILCP are hardly defined. Due to the limited and precious samples, the precise progenitor-progeny relationship of these progenitor/precursors warrants further investigation. The fetal ILCPs also showed intestine-biased feature, consistent with the localdifferentiation characteristics of ILCs. In addition to ILCPS, the precursors of individual ILC subsets were identified in our study, and several common features shared by these precursors were revealed. For instance, the expression level of the featured genes of each ILC subset was lower in the precursors than in mature ILCs, and more precursors were detected at $8 \mathrm{PCW}$ than 10 and $12 \mathrm{PCW}$. More importantly, despite the potential heterogeneity of these cells, majority of the precursors were proliferative and expressed DNA replication-related genes. In contrast, the ILCPs and mature ILCs were quiescent. The similar dynamics of cell proliferation is also found in the T cell development that involves multiple runs of cell proliferation. In the initial steps of T cell development, the most primitive ETPs in thymus are quiescent, then the ETPs start to proliferate, simultaneously commit to $T$ cell lineage. ${ }^{46,59,62}$ Given the similarity of T cell and ILC development, it was therefore likely that ILC precursors might proliferate when committed to individual ILC lineage, and the final step of ILC maturation might not involve cell expansion. Potential markers to distinguish each ILC precursors have been also provided, however, ideal surface marker to perfectly differ Pre-ILC3 from ILCP is to be identified and moreover, functional validation needs further investigation.

The heterogeneity and tissue distribution of individual ILC populations have been studied in the adult humans and mice, showing the varied phenotypes and functions of ILCS in different tissues. Our data also suggested the tissue-preferred distribution of ILC subsets with distinct molecular features. For example, CXCR4 expressed in ILC1_b cells might be involved in the migration of these cells into skin. ILC_1d cells were heterogenous, containing ILC1-biased and NK-biased cells. Although the ILC1-biased and NK-biased cells could be distinguished by lineage-specific feature genes, their lineage bifurcation was not obvious in the trajectory analysis. They were possibly relatively immature at this early fetal stage which could develop into mature CD56 ${ }^{+}$ILC1 cells and NK cells, respectively. ${ }^{1,43}$ Moreover, CCR9-expressing ILC2 subset was mainly located in thymus, consistent with the function of CCR9-CCL25 in ETP settling in thymus. Notably, this subset of ILC2 was $\mathrm{CRTH}_{2}^{-}$and $\mathrm{CD} 117^{-}$, suggestive of the unconventional ILC2s identity since these cells had the comparable GATA3 but low RORyt levels compared with the conventional ILC2s and ILC3s, respectively. Notably, the chemokine receptor CCR9 is also highly expressed on ILC2P ( Lin $^{-} \mathrm{SCa}-1^{\text {hi }}$ ID2 ${ }^{\text {hi }} \mathrm{GA}$ $\mathrm{TA} 3^{\mathrm{hi}} \mathrm{KLRG}^{-}$) and mature ILC2 in bone marrow and small intestine of adult mouse. Significant reduction of GATA $3^{\text {hi }}$ ILCS, including ILC2 and their progenitors, is observed in CCR9-deficient mice, especially in the intestine, suggestive of an important role of CCR9 in the recruitment of ILC2P to intestine. ${ }^{34}$ Besides, CCR9 expression was also reported on mouse fetal mesentery ILC2 progenitors and mature ILC2. ${ }^{108}$ Another CCR6- and KIT-expressing ILC2 subset without expression of CRTH2 was speculated to be the fetal counterpart of the recently reported c$\mathrm{Kit}^{\text {hi }} \mathrm{CCR6}^{+}$ILC2s in the adult, harboring ILC2-ILC3 plasticity.

In summary, we have generated a high-resolution single-cell transcriptomic dataset that yields detailed information about the development and heterogeneity of human ILCS and related progenitor/precursors at fetal stage, as well as their transcriptional landscape and immunophenotypes, especially for the novel ILC subsets.

\section{MATERIALS AND METHODS Human samples}

Healthy human embryonic and fetal samples were obtained following the elective medical termination of pregnancy at the Academy of Military Medical Sciences (the Fifth Medical Center of the PLA General Hospital). All experiments were performed in accordance with protocols approved by the Ethics Committee of the Affiliated Hospital of Academy of Military Medical Sciences (ky-2020-8-19), and local and state ethical guidelines and principles. The written informed consent was obtained before sample collection. The integrity of the samples has been confirmed and examined at multiple steps. From the beginning, the Obstetrics and Gynecology clinicians confirmed the collected embryos and fetus that were free of any known genetic or developmental abnormality. Before sample processing, the morphological examinations were performed to exclude samples with any potential developmental defects. Moreover, flow-cytometry analysis was used to examine whether the cellular profile of collected tissue was consistent with literature. Samples were classified according to the standard crown-rump length (CRL) measurement and were determined. ${ }^{109,110}$ Samples used in this study were from 8 to 12 PCW, with weeks indicating weeks post-fertilization. The gender of samples used for scRNA-seq was determined based on the expression of XIST and RPS4Y1 latterly. All the information of sample was summarized in Supplementary information, Fig. S1.

\section{Cell lines}

OP9-DL4 (OP9 stromal line engineered to express human Delta-like-4 ligand) (Mus musculus, a generous gift from Dr. Nengming Xiao, Xiamen University) were maintained at $37{ }^{\circ} \mathrm{C}$ with $5 \% \mathrm{CO}_{2}$ in a-MEM (GIBCO, 12561056) supplemented with $20 \%$ Fetal Bovine Serum (FBS) (HyClone, SH30070.03), penicillin-streptomycin (GIBCO, 15140-122), $50 \mu \mathrm{M}$ 2mercaptoethanol (GIBCO, 21985-023) and L-glutamine.

\section{Flow cytometry}

Cells were stained by the following antibodies: BV421 anti-human CD45 (BD, Cat\#563879), APC-Cy7 anti-human CD3 (Biolegend, Cat\#300426), APC-Cy7 antihuman CD5 (BD Cat\#563516), APC-Cy7 anti-human CD11c (Biolegend, 
Cat\#337218), APC-Cy7 anti-human CD14 (Biolegend, Cat\#325620), APC-Cy7 anti-human CD19 (BD, Cat\#560177), APC-Cy7 anti-human FcERla, (Biolegend, Cat\#334632), APC anti-human ITGB7 (Biolegend, Cat\#321207), APC anti-human CCR7 (Biolegend, Cat\#353213), APC anti-human CCR9 (Biolegend, Cat\#358907), BV605 anti-human CD117 (Invitrogen, Cat\#313218), FITC anti-human CD34 (BD, Cat\#555821), PE-Cy7 anti-human CD127 (eBioscience Cat\#25-1278-41), Alexa Fluor 647 anti-human CRTH2 (BD, Cat\#558042), FITC anti-human CRTH2 (Biolegend, Cat\#350108), FITC anti-human CD3 (Biolegend, Cat\#300406), FITC anti-human CD5 (Biolegend, Cat\#300606), FITC anti-human CD14 (Biolegend, Cat\#301804), FITC anti-human CD19 (Biolegend, Cat\#302256), FITC anti-human CD11c (BD, Cat\#561355), FITC anti-human FceRla (Biolegend, Cat\#334640), PE anti-human CD161 (Biolegend, Cat\#339903), APC-Cy7 anti-human CD45 (BD, Cat\#557833), Percp anti-human CD56 (Biolegend, Cat\#362525), PE-Cy7 antihuman IFN-g (BD, Cat\#502528), BV421 anti-human IL13 (BD, Cat\#563580), APC anti-human IL17a (BD, Cat\#512333), BV510 anti-human CD19 (BD, Cat\#562947), APC-Cy7 anti-human CD56 (Biolegend, Cat\#362512), APC anti-human CD33 (Biolegend, Cat\#366606), PE anti-human CD4 (Biolegend, Cat\#300507), PE-Cy7 anti-human CD8 (BD, Cat\#557746), BV510 anti-human CD7 (BD, Cat\#563650), TotalSeq ${ }^{\text {TM }}$-B0251 anti-human Hashtag 1 Antibody (Biolegend, Cat\#394631), TotalSeq ${ }^{\mathrm{TM}}$-B0252 anti-human Hashtag 2 Antibody (Biolegend, Cat\#394633), TotalSeq ${ }^{\mathrm{TM}}$-B0253 anti-human Hashtag 3 Antibody (Biolegend, Cat\#394635), TotalSeq ${ }^{\text {TM }}$-B0254 anti-human Hashtag 4 Antibody (Biolegend, Cat\#394637), TotalSeq ${ }^{\mathrm{TM}}$-B0255 anti-human Hashtag 5 Antibody (Biolegend, Cat\#394639), TotalSeq ${ }^{\text {TM }}$-B0256 anti-human Hashtag 6 Antibody (Biolegend,Cat\#394641)

\section{Preparation of single-cell suspensions}

Fetal tissues including lung, intestine, skin, spleen and thymus were isolated and transferred to RPMI1640 medium (Gibco, 11875093) containing 10\% FBS (HyClone, SH30070.03) on ice. Then, the tissues were washed with PBS for 3 times and transferred to pre-warmed digestion medium containing $100 \mu \mathrm{g} / \mathrm{mL}$ DNase I (Sigma-Aldrich, DN25) and $0.1 \mathrm{~g} / \mathrm{mL}$ Collagenase I (Sigma, C2674) in PBS containing $10 \%$ FBS. The amount of enzyme depended on the size of tissues, such as $1-2 \mathrm{~mL}$ enzyme mixture for week 8 samples and $4-6 \mathrm{~mL}$ enzyme mixture for week 10 to 12 samples. Tissues were shaken vigorously for $30 \mathrm{~s}$ and further incubated at $37^{\circ} \mathrm{C}$ for $30-40 \mathrm{~min}$ in incubator with general shaking every $6 \mathrm{~min}$ to release cells. Released cells passed through a $70 \mu \mathrm{m}$ cell strainer (BD, 352350) and were collected in $15 \mathrm{~mL}$ tubes containing $8 \mathrm{~mL}$ FACSbuffer to neutralize enzymes. Cells were then collected by spinning at $500 \times g$ for $5 \mathrm{~min}$, and suspended in FACS-buffer (1× PBS with 1\% BSA) for subsequent staining.

Fetal liver was mechanically dissociated into single-cell suspension using scalpels and syringes. Cells were then filtered through a $70 \mu \mathrm{m}$ cell strainer (BD, 352350) and collected by spinning at $400 \times g$ for $6 \mathrm{~min}$. Resuspend in RBC lysis buffer (BD, 555899) for about 8 min to remove erythrocytes. After neutralization, remaining cells were collected in FACS buffer (1× PBS with $1 \% \mathrm{BSA}$ ) for subsequent staining.

\section{FACS and cell hashing for scRNA-seq}

1-2 million cells from each tissue were resuspended in $50 \mu \mathrm{L}$ Cell Staining Buffer (Biolegend, 420201) and $5 \mu \mathrm{L}$ of Human TruStain FCX'M Fc Blocking reagent (Biolegend, 422301) was added and incubate for $10 \mathrm{~min}$ at $4^{\circ} \mathrm{C}$. Antibody pool of each hashtag antibody with titrated doseage $(0.2 \mu \mathrm{L}$ for 1 million cells), and sorting antibodies were prepared and added to the cell suspension, and incubated for $30 \mathrm{~min}$ at $4{ }^{\circ} \mathrm{C}$. After washing, staining with 7$A A D$ was performed for about $5 \mathrm{~min}$. Cells were sorted with gating strategy shown in Supplementary information, Fig. S1. on BD FACS Aria II and harvested in $200 \mu \mathrm{L}$ PCR tube. Cells from each tissue were pooled into one $1.5 \mathrm{~mL}$ centrifuge tube. Cells were collected at $400 \times g$ at $4{ }^{\circ} \mathrm{C}$ for $5 \mathrm{~min}$, resuspend in 50 uL FACS-buffer (1X PBS with $1 \%$ BSA) to load into one lane of a 10x Chromium V3 chip. The CDNA preparation was performed following the instruction manual, and the hashtag library was prepared following the BioLegend TotalseqB guide. The final CDNA library and tag library were both sequenced on NovaSeq 6000. Cells were sequenced to an average depth of $0.1 \mathrm{M}$ per cell for cDNA and $0.01 \mathrm{M}$ per cell for hashtags.

\section{Coculture for assessment of B, T, NK and myeloid lineage potential at bulk and single-cell levels}

Co-cultivation on the OP9 stromal line engineered to express human Delta-like-4 ligand (OP9-DL4, a generous gift from Dr. Nengming Xiao, Xiamen University) and OP9 stromal cells with SCF, FL, IL-7 and IL-15 was used to test the $B, T$, NK and myeloid lineage potential of $\mathrm{Lin}^{-} \mathrm{CD} 45^{+} \mathrm{CD} 127^{+} \mathrm{CD} 34^{+} \mathrm{IL}-3 \mathrm{RA}^{+/-}$progenitors. For bulk culture, $1-1.5 \times$ $10^{4}$ stromal cells were pre-seeded in 24-well round bottom plates one night before culture. Freshly FACS-sorted progenitors (50-200 cells) were seeded onto established, nonirradiated OP9-DL4 or OP9 stromal cells. For single-cell culture, $3-5 \times 10^{3}$ stromal cells were pre-seeded in 96-well plates one night before culture. $\mathrm{Lin}^{-} \mathrm{CD} 45^{+} \mathrm{CD} 127^{+} \mathrm{CD} 34^{+} \mathrm{IL}$ $3 \mathrm{RA}^{+}$progenitors were sorted in single-cell mode and then plated on the stromal cells. Cells were cultured in a-MEM (GIBCO, 12561-056) containing 20\% Fetal Bovine Serum (HyClone, SH30070.03), $50 \mu \mathrm{M} 2-$ mercaptoethanol (GIBCO, 21985-023), penicillin-streptomycin (GIBCO, $15140-122)$, L-glutamine in the presence of SCF $(5 \mathrm{ng} / \mathrm{mL}), \mathrm{IL}-7$ ( $5 \mathrm{ng} /$ $\mathrm{mL}$ ), Flt3 ligand $(5 \mathrm{ng} / \mathrm{mL})$ and IL-15 $(10 \mathrm{ng} / \mathrm{mL})$ (cytokines from PeproTECH) for 8-14 days. Stromal cells were passaged when the confluence reached about $80 \%-95 \%$. Co-cultured cells were separated by $0.25 \%$ Trypsin-EDTA (Sigma, $59428 \mathrm{C}$ ) solution and collected by spinning at $500 \times \mathrm{g}$ for $5 \mathrm{~min}$ at $4{ }^{\circ} \mathrm{C}$. All cultures were analyzed in parallel with negative control wells that contained OP9-DL4 or OP9 stromal cells and identical culture medium without progenitors. T, B, NK and myeloid cells were identified as positive for its respective lineage markers and negative for other lineage markers. For clonal assay, clones were required to have over ten $C D 45^{+}$cell-gated events (of the appropriate cell-surface phenotype) to be considered positive.

\section{Coculture for assessment of ILC lineage potential at bulk and single-cell levels}

For bulk culture, 1-1.5 × $10^{4}$ OP9-DL4 stromal cells were pre-seeded in 24well round bottom plates one night before co-culture. Freshly FACS-sorted $\mathrm{Lin}^{-} \mathrm{CD} 45^{+} \mathrm{CD} 127^{+} \mathrm{CD}^{+} 4^{+} \mathrm{IL}-3 \mathrm{RA}{ }^{+/}$progenitors $(150$ cells $)$ or ILCP $\left(\mathrm{Lin}^{-} \mathrm{CD} 45^{+} \mathrm{CD}^{+} \mathrm{CD} 127^{+} \mathrm{CD} 117^{+}\right)$were seeded into the wells with preseeded OP9-DL4 stromal cells. For single-cell culture, 3-5 × $10^{3}$ OP9-DL4 stromal cells were pre-seeded in 96-well round bottom plates one night before co-culture. Cells of $\mathrm{Lin}^{-} \mathrm{CD} 45^{+} \mathrm{CD} 127^{+} \mathrm{CD} 34^{+} \mathrm{IL}-3 \mathrm{RA}{ }^{+}$progenitors were sorted in single-cell mode and then plated on the stromal cells. Cells were cultured in Yssel's medium supplemented with $2 \%$ human $A B$ serum (GeminiBio, 100-512) as Dr. James P. Di Santo's group described in the presence of IL-2, IL-7, IL-1 $\beta, \quad \mathrm{IL}-23, \mathrm{IL}-25$ and IL-33 (PeproTECH) for 10-14 days. ${ }^{36}$ Stromal cells were passaged when the confluence reached about $80 \%-95 \%$. Co-cultured cells were treated by $0.25 \%$ Trypsin-EDTA (Sigma, $59428 \mathrm{C}$ ) solution and collected by spinning at $500 \times g$ for $5 \mathrm{~min}$ at $4^{\circ}$ C. All cultures were analyzed in parallel with negative control wells that contained OP9-DL4 stromal and identical culture medium without progenitors. To detect the cytokine expression, cells were stimulated with cell stimulation cocktail (Tonbo, TNB-4975), $2 \mu \mathrm{L}$ per sample, for $4 \mathrm{~h}$. Intracellular staining of cytokines was performed with Cytofix/Cytoperm Kit (BD, 554714).

\section{Yssel's medium}

Yssel's medium was prepared in house by using IMDM (Invitrogen, 31980030) plus $0.25 \%$ (w/v) BSA (Sigma, A9647), $1.8 \mathrm{mg} / \mathrm{L}$ 2-amino ethanol, $40 \mathrm{mg} / \mathrm{L}$ Apo-transferrin, $5 \mathrm{mg} / \mathrm{L}$ insulin and Penicillin/Streptomycin.

\section{Sequencing and pre-processing data}

Single-cell RNA-seq library was prepared and performed on the Chromium platform (10x Genomics), using the single cell expression $3^{\prime}$ profiling chemistry combined with cell hashing. Cell-hashing antibody (HTO)labeled cells from 2-3 tissues were pooled together and washed with RPMI-1640 immediately before loading on the 10x controller. Then the library was constructed following the manufacturer's protocol, with some additional steps for the amplification of HTO barcodes. The raw data were aligned to the hg19 reference genome using cellranger count (Version 3.0), and the hashed cells were demultiplexed using HTODemux function in Seurat 3 , the cells aligned to doublet and negative group were removed from further analysis.

Single-cell data analysis (including quality control, data normalization, dimension reduction, clusters detection) were performed using Seurat 3 in R. Firstly, count matrices of gene expression derived from the cellranger output were subjected to Seurat. Low quality cells (less than 1000 genes, less than 1000 UMls or less than 20 percent UMls mapping on mitochondria in one cell) were filtered before data normalization was performed. The filted data were integrated using method based on identification of 'anchors' between pairs of datasets following the tutorial at https://satijalab.org/seurat/v3.1/integration.html. Finally, PCA (principal component analysis) was performed and significant PCs were selected on the basis of the elbow of standard deviations of PCs. Next, UMAP, $t$-SNE, trimap, clustering analysis and re-clustering analysis were complemented using selected PCs using Seurat $3^{111}$ and Scanpy. ${ }^{112}$ 


\section{PAGA analysis}

We quantified the connectivity of cell clusters using the PAGA method, ${ }^{113}$ using single-cell analysis package Scanpy based on significant PCs.

\section{SCENIC analysis}

The transcriptional and regulatory characteristics of the different progenitors including HSPC, LP1, LP2, ILCP, ETP, pre pro-B populations, gene regulatory network analysis was performed using SCENIC. ${ }^{114}$ First, regulatory modules (regulons) were inferred from co-expression and DNA motif analysis. These regulons were then evaluated in each cell to ascertain their activity before a binary matrix was obtained. To profile the gene regulatory module features of all progenitors, the Spearman correlation coefficient between regulons was calculated, and only regulons with a correlation coefficient larger than 0.3 with at least one other regulon and activated in at least $30 \%$ cells in any progenitor clusters were included for visualization.

\section{STEMNET analysis}

In order to infer the developmental potential of progenitor populations, STEMNET analysis was implemented. ${ }^{115}$ Firstly, we defined the most mature cells, such as B-cells, T-cells, ILCs, monocytes, macrophages, erythrocytes, megakaryocytes, and mast cells in our data as mature subsets (given that pre-pDCs in our data were immature, the mature pDCs in bone marrow $^{69}$ were used as pDC subsets), allowing the STEMNET algorithm to calculate posterior probabilities of all the progenitor populations based on the highly expressed genes with average expression $>0.1$.

\section{Gene expression network construction}

To construct TFs correlation network during ILC specification, the differently expressed TF-encoding genes of each cluster were calculated and correlations between TFs were obtained using Pearson correlation. Then, only TFs with positive correlation with more than 3 other genes were retained for visualization using Cytoscape (https://cytoscape.org/).

\section{DEGs detection and cluster biomarker identification}

DEGs were identified by running the 'FindAllMarkers' function in Seurat using the 'Wilcox test'. All DEGs of specific clusters were listed in supplementary tables. Surface markers and transcription factors list were downloaded from Cell Surface Protein Atlas (http://wlab.ethz.ch/cspa/) and HumanTFDB3.0 (http://bioinfo.life.hust.edu.cn/HumanTFDB/).

\section{Cell cycle analysis}

For cell cycle analysis, cell cycle-related genes consisting of a previously defined core set of $43 \mathrm{G} 1 / \mathrm{S}$ genes and $54 \mathrm{G} 2 / \mathrm{M}$ genes were used, which were listed in Supplementary information, Table S6. We used a way similar to that reported in Embryonic endothelial evolution towards first hematopoietic stem cells revealed by single-cell transcriptomic and functional analyses to classify the cycling phases of the cells. ${ }^{116}$ We calculated the average expression of each gene set as corresponding scores, and manually assigned cells to approximate cell cycle phases based on the scores.

\section{Trajectory analysis}

We performed cellular trajectory reconstruction analysis using CytoTRACE Kernel of CellRank. ${ }^{117}$ Firstly, the KNN graph containing information about the undirected connectivities among cells was constructed. Then, a pseudotemporal ordering of cells was calculated using CytoTRACE, ${ }^{118}$ an algorithm that predicts cellular plasticity states based on the transcriptional diversity. Lastly, a transition matrix was constructed based on the KNN graph and pseudotemporal ordering and projected onto umap plot.

\section{MATERIAL AVAILABILITY}

Further information and requests for resources and reagents should be directed to and will be fulfilled by the Lead Contact Dr. Bing Liu (bingliu17@yahoo.com). The scRNA-seq data of our study have been deposited at GEO (NCBI) with accession code GSE163587. This study did not generate new unique reagents.

\section{REFERENCES}

1. Meininger, I. et al. Tissue-specific features of innate lymphoid cells. Trends Immunol. 41, 902-917 (2020).
2. Vivier, E. et al. Innate lymphoid cells: 10 years on. Cell 174, 1054-1066 (2018).

3. Hazenberg, M. \& Spits, H. Human innate lymphoid cells. Blood 124, 700-709 (2014).

4. Miller, M. \& Reinhardt, R. The heterogeneity, origins, and impact of migratory ilLC2 cells in anti-helminth immunity. Front. Immunol. 11, 1594 (2020).

5. Bernink, J. H. et al. Human type 1 innate lymphoid cells accumulate in inflamed mucosal tissues. Nat. Immunol. 14, 221-229 (2013).

6. Rao, A. et al. Cytokines regulate the antigen-presenting characteristics of human circulating and tissue-resident intestinal ILCs. Nat. Commun. 11, 2049 (2020).

7. Spits, H. et al. Innate lymphoid cells-a proposal for uniform nomenclature. Nat. Rev. Immunol. 13, 145-149 (2013).

8. Cupedo, T. et al. Human fetal lymphoid tissue-inducer cells are interleukin 17producing precursors to RORC+ CD127+ natural killer-like cell. Nat. Immunol. 10, 66-74 (2009).

9. Mebius, R., Rennert, P. \& Weissman, I. Developing lymph nodes collect CD4 +CD3- LTbeta+ cells that can differentiate to APC, NK cells, and follicular cells but not T or B cells. Immunity 7 493-504 (1997).

10. Colonna, M. Interleukin-22-producing natural killer cells and lymphoid tissue inducer-like cells in mucosal immunity. Immunity 31, 15-23 (2009).

11. Cella, M. et al. A human natural killer cell subset provides an innate source of IL22 for mucosal immunity. Nature 457, 722-725 (2009).

12. Tait Wojno, E. \& Artis, D. Emerging concepts and future challenges in innate lymphoid cell biology. J. Exp. Med. 213, 2229-2248 (2016).

13. Yang, Q. \& Bhandoola, A. The development of adult innate lymphoid cells. Curr. Opin. Immunol. 39, 114-120 (2016).

14. Kondo, M., Weissman, I. \& Akashi, K. Identification of clonogenic common lymphoid progenitors in mouse bone marrow. Cell 91, 661-672 (1997).

15. Galy, A., Travis, M., Cen, D. \& Chen, B. Human T, B, natural killer, and dendritic cells arise from a common bone marrow progenitor cell subset. Immunity $\mathbf{3}$, 459-473 (1995).

16. Cumano, A. et al. New molecular insights into immune cell development. Ann. Rev. Immunol. 37, 497-519 (2019).

17. Zook, E. C. \& Kee, B. L. Development of innate lymphoid cells. Nat. Immunol. 17, 775-782 (2016).

18. Karamitros, D. et al. Single-cell analysis reveals the continuum of human lympho-myeloid progenitor cells. Nat. Immunol. 19, 85-97 (2018).

19. Harly, C. et al. The transcription factor TCF-1 enforces commitment to the innate lymphoid cell lineage. Nat. Immunol. 20, 1150-1160 (2019).

20. Scoville, S., Freud, A. \& Caligiuri, M. Modeling human natural killer cell development in the Era of innate lymphoid cells. Front. Immunol. 8, 360 (2017).

21. Scoville, S. D. et al. A progenitor cell expressing transcription factor RORyt generates all human innate lymphoid cell subsets. Immunity 44, 1140-1150 (2016).

22. Freud, A. G. et al. Evidence for discrete stages of human natural killer cell differentiation in vivo. J. Exp. Med. 203, 1033-1043 (2006).

23. McFarland, A. \& Colonna, M. Sense and immuno-sensibility: innate lymphoid cell niches and circuits. Curr. Opin. Immunol. 62, 9-14 (2020).

24. Simoni, Y. et al. Human innate lymphoid cell subsets possess tissue-type based heterogeneity in phenotype and frequency. Immunity 46, 148-161 (2017).

25. Xu, W. et al. An Id2-reporter mouse redefines innate lymphoid cell precursor potentials. Immunity 50, 1054-1068 (2019).

26. $\mathrm{Yu}, \mathrm{Y}$. et al. The transcription factor $\mathrm{Bcl} 11 \mathrm{~b}$ is specifically expressed in group 2 innate lymphoid cells and is essential for their development. J. Exp. Med. 212, 865-874 (2015).

27. $\mathrm{Yu}, \mathrm{X}$. et al. The basic leucine zipper transcription factor NFIL3 directs the development of a common innate lymphoid cell precursor. elife 3, e04406 (2014).

28. Tindemans, I., Serafini, N., Di Santo, J. \& Hendriks, R. GATA-3 function in innate and adaptive immunity. Immunity 41, 191-206 (2014).

29. Constantinides, M., McDonald, B., Verhoef, P. \& Bendelac, A. A committed precursor to innate lymphoid cells. Nature 508, 397-401 (2014).

30. Montaldo, E. et al. Human RORyt $(+) \mathrm{CD} 34(+)$ cells are lineage-specified progenitors of group 3 RORyt(+) innate lymphoid cells. Immunity 41, 988-1000 (2014).

31. Cervantes-Barragan, L. \& Colonna, M. Chemical sensing in development and function of intestinal lymphocytes. Curr. Opin. Immunol. 50, 112-116 (2018).

32. Serafini, N., Vosshenrich, C. \& Di Santo, J. Transcriptional regulation of innate lymphoid cell fate. Nat. Rev. Immunol. 15, 415-428 (2015).

33. Klose, C. et al. Differentiation of type $1 \mathrm{ILCs}$ from a common progenitor to all helper-like innate lymphoid cell lineages. Cell 157, 340-356 (2014).

34. Hoyler, T. et al. The transcription factor GATA-3 controls cell fate and maintenance of type 2 innate lymphoid cells. Immunity 37, 634-648 (2012).

35. Yang, Q. et al. TCF-1 upregulation identifies early innate lymphoid progenitors in the bone marrow. Nat. Immunol. 16, 1044-1050 (2015). 
36. Lim, A. I. et al. Systemic human ILC precursors provide a substrate foR Tissue ILC differentiation. Cell 168, 1086-1100 (2017).

37. Mjösberg, J. \& Mazzurana, L. ILC-poiesis: making tissue ILCs from blood. Immunity 46, 344-346 (2017).

38. Nagasawa, M. et al. KLRG1 and NKp46 discriminate subpopulations of human CD117CRTH2 ILCs biased toward ILC2 or ILC3. J. Exp. Med. 216, 1762-1776 (2019).

39. Chen, L. et al. CD56 expression marks human group 2 innate lymphoid cell divergence from a shared NK cell and group 3 innate lymphoid cell developmental pathway. Immunity 49, 464-476 (2018).

40. Li, N. et al. Mass cytometry reveals innate lymphoid cell differentiation pathways in the human fetal intestine. J. Exp. Med. 215, 1383-1396 (2018).

41. Bar-Ephraim, Y. E. et al. CD62L is a functional and phenotypic marker for circulating innate lymphoid cell precursors. J. Immunol. 202, 171-182 (2019).

42. Simoni, Y. \& Newell, E. Dissecting human ILC heterogeneity: more than just three subsets. Immunology 153, 297-303 (2018).

43. Yudanin, N. A. et al. Spatial and temporal mapping of human innate lymphoid cells reveals elements of tissue specificity. Immunity 50, 505-519 (2019).

44. Björklund, Å. K. et al. The heterogeneity of human $C D 127(+)$ innate lymphoid cells revealed by single-cell RNA sequencing. Nat. Immunol. 17, 451-460 (2016).

45. Mazzurana, L. et al. Tissue-specific transcriptional imprinting and heterogeneity in human innate lymphoid cells revealed by full-length single-cell RNAsequencing. Cell Res. 31, 554-568 (2021).

46. Zeng, Y. et al. Single-cell RNA sequencing resolves spatiotemporal development of pre-thymic lymphoid progenitors and thymus organogenesis in human embryos. Immunity 51, 930-948 (2019).

47. Kernfeld, E. M. et al. A single-cell transcriptomic atlas of thymus organogenesis resolves cell types and developmental maturation. Immunity 48, 1258-1270 (2018).

48. Cupedo, T. ILC2: at home in the thymus. Eur. J. Immunol. 48, 1441-1444 (2018).

49. Rossi, S. et al. RANK signals from CD4(+)3(-) inducer cells regulate development of Aire-expressing epithelial cells in the thymic medulla. J. Exp. Med. 204, 1267-1272 (2007).

50. Jones, R. et al. Dynamic changes in intrathymic ILC populations during murine neonatal development. Eur. J. Immunol. 48, 1481-1491 (2018).

51. Gentek, R. et al. Modulation of signal strength switches notch from an inducer of T cells to an inducer of ILC2. Front. Immunol. 4, 334 (2013).

52. Qian, L., Bajana, S., Georgescu, C., Peng, V. \& Sun, X. H. Suppression of ILC2 differentiation from committed $T$ cell precursors by $E$ protein transcription factors. J. Exp. Med. 216, 884-899 (2019).

53. Nagasawa, M., Germar, K., Blom, B. \& Spits, H. Human CD5 innate lymphoid cells are functionally immature and their development from CD34 progenitor cells is regulated byld2. Front. Immunol. 8, 1047 (2017).

54. Wang, H. C., Qian, L., Zhao, Y., Mengarelli, J. \& Sun, X. H. Downregulation of E protein activity augments an ILC2 differentiation program in the thymus. $J$. Immunol. 198, 3149-3156 (2017).

55. Hoorweg, K. et al. Functional differences between human NKp44(-) and NKp44(+) RORC(+) innate lymphoid cells. Front. Immunol. 3, 72 (2012).

56. Forkel, M. et al. Composition and functionality of the intrahepatic innate lymphoid cell-compartment in human nonfibrotic and fibrotic livers. Eur J Immunol. 47, 1280-1294 (2017).

57. Miller, D., Motomura, K., Garcia-Flores, V., Romero, R. \& Gomez-Lopez, N. Innate lymphoid cells in the maternal and fetal compartments. Front. Immunol. 9, 2396 (2018).

58. Popescu, D. M. et al. Decoding human fetal liver haematopoiesis. Nature $\mathbf{5 7 4}$ 365-371 (2019).

59. Lavaert, M. et al. Integrated scRNA-Seq identifies human postnatal thymus seeding progenitors and regulatory dynamics of differentiating immature thymocytes. Immunity 52, 1088-1104 (2020).

60. Le, J. et al. Single-Cell RNA-Seq mapping of human thymopoiesis reveals lineage specification trajectories and a commitment spectrum in $\mathrm{T}$ cell development. Immunity 52, 1105-1118 (2020).

61. Zhou, W. et al. Single-cell analysis reveals regulatory gene expression dynamics leading to lineage commitment in early T cell development. Cell Syst. 9, 321-337 (2019).

62. Park, J. E. et al. A cell atlas of human thymic development defines T cell repertoire formation. Science 367, 6480 (2020).

63. Gury-BenAri, M. et al. The spectrum and regulatory landscape of intestinal innate lymphoid cells are shaped by the microbiome. Cell 166, 1231-1246 (2016).

64. Mjösberg, J. M. et al. Human IL-25- and IL-33-responsive type 2 innate lymphoid cells are defined by expression of CRTH2 and CD161-1062. Nature Immunol. 12, 1055-1062 (2011). 12.

65. Rosmaraki, E. E. et al. Identification of committed NK cell progenitors in adult murine bone marrow. Eur. J. Immunol. 31, 1900-1909 (2001).
66. Carotta, S., Pang, S., Nutt, S. \& Belz, G. Identification of the earliest NK-cell precursor in the mouse BM. Blood 117, 5449-5452 (2011).

67. Vosshenrich, C. \& Di Santo, J. Developmental programming of natural killer and innate lymphoid cells. Curr. Opin. Immunol. 25, 130-138 (2013).

68. Komorowska, K. et al. Hepatic leukemia factor maintains quiescence of hematopoietic stem cells and protects the stem cell pool during regeneration. Cell Rep. 21, 3514-3523 (2017).

69. Cytlak, U. et al. Differential IRF8 transcription factor requirement defines two pathways of dendritic cell development in humans. Immunity 53, 353-370 (2020).

70. Lai, C. \& Mager, D. Role of runt-related transcription factor 3 (RUNX3) in transcription regulation of natural cytotoxicity receptor 1 (NCR1/NKp46), an activating natural killer (NK) cell receptor. J. Biol. Chem. 287, 7324-7334 (2012).

71. Ebihara, T. et al. Runx3 specifies lineage commitment of innate lymphoid cells. Nat. Immunol. 16, 1124-1133 (2015).

72. Burger, J. A., Zvaifler, N. J., Tsukada, N., Firestein, G. S. \& Kipps, T. J. Fibroblast-like synoviocytes support B-cell pseudoemperipolesis via a stromal cell-derived factor-1- and CD106 (VCAM-1)-dependent mechanism. J. Clin. Invest. 107, 305-315 (2001).

73. Quan, C. et al. Dermal fibroblast expression of stromal cell-derived factor-1 (SDF1) promotes epidermal keratinocyte proliferation in normal and diseased skin. Protein Cell. 6, 890-903 (2015).

74. Wyles, J. P., Wu, Z., Mirski, S. E. \& Cole, S. P. Nuclear interactions of topoisomerase II alpha and beta with phospholipid scramblase 1. Nucleic Acids Res. 35, 4076-4085 (2007).

75. Maric, J. et al. Cytokine-induced endogenous production of prostaglandin $D$ is essential for human group 2 innate lymphoid cell activation. The J. Allergy Clin. Immunol. 143, 2202-2214.e5 (2019).

76. Dahlgren, M. W. et al. Adventitial stromal cells define group. 2 innate lymphoid cell tissue niches. Immunity 50, 707-722.e6 (2019).

77. Bernink, J. H., Ohne, Y., Teunissen, M., Wang, J. \& Wu, J. Krabbendam L, et al. cKit-positive ILC2s exhibit an ILC3-like signature that may contribute to IL-17mediated pathologies. Nat. Immunol. 20, 992-1003 (2019).

78. Hochdörfer, T., Winkler, C., Pardali, K. \& Mjösberg, J. Expression of c-Kit discriminates between two functionally distinct subsets of human type 2 innate lymphoid cells. Eur. J. Immunol. 49, 884-893 (2019).

79. Li, L., Leid, M. \& Rothenberg, E. V. An early T cell lineage commitment checkpoint dependent on the transcription factor Bcl11b. Science 329, 89-93 (2010).

80. Califano, D. et al. Transcription factor Bcl11b controls identity and function of mature type 2 innate lymphoid cells. Immunity 43, 354-368 (2015).

81. Kostrzewski, T. \& Brady, H. ILC lineage specification: to be or not $11 \mathrm{~b}$, that is the question. Immunity 43, 220-222 (2015).

82. Schwarz, B. A. et al. Selective thymus settling regulated by cytokine and chemokine receptors. J. Immunol. 178, 2008-2017 (2007).

83. Liu, C. et al. Coordination between CCR7- and CCR9-mediated chemokine signals in prevascular fetal thymus colonization. Blood 108, 2531-2539 (2006).

84. Zlotoff, D. A. et al. and CCR9 together recruit hematopoietic progenitors to the adult thymus. Blood 115, 1897-1905 (2010).

85. Mazzurana, L. et al. Suppression of Aiolos and Ikaros expression by lenalidomide reduces human ILC3-ILC1/NK cell transdifferentiation. Eur. J. Immunol. 49, 1344-1355 (2019)

86. Shikhagaie, M. M. et al. Neuropilin-1 Is expressed on lymphoid tissue residing LTi-like group 3 innate lymphoid cells and associated with ectopic lymphoid aggregates. Cell Rep. 18, 1761-1773 (2017).

87. Fuchs, A., Cella, M., Giurisato, E., Shaw, A. \& Colonna, M. Cutting edge: CD96 (tactile) promotes NK cell-target cell adhesion by interacting with the poliovirus receptor (CD155). J. Immunol. 172, 3994-3998 (2004).

88. Cepek, K. L. et al. Adhesion between epithelial cells and T lymphocytes mediated by E-cadherin and the alpha E beta 7 integrin. Nature 372, 190-193 (1994).

89. Ishizuka, I. E. et al. Single-cell analysis defines the divergence between the innate lymphoid cell lineage and lymphoid tissue-inducer cell lineage. Nat. Immunol. 17, 269-276 (2016).

90. Montaldo, E., Juelke, K. \& Romagnani, C. Group 3 innate lymphoid cells (ILC3s): origin, differentiation, and plasticity in humans and mice. Eur. J. Immunol. 45, 2171-2182 (2015).

91. Weber, B. N. et al. A critical role for TCF-1 in T-lineage specification and differentiation. Nature 476, 63-68 (2012).

92. Yang, Q. et al. T cell factor 1 is required for group 2 innate lymphoid cell generation. Immunity 38, 694-704 (2013).

93. Held, W., Clevers, H. \& Grosschedl, R. Redundant functions of TCF-1 and LEF-1 during $T$ and NK cell development, but unique role of TCF-1 for Ly49 NK cell receptor acquisition. Eur. J. Immunol. 33, 1393-1398 (2010).

94. Ioannidis, V., Kunz, B., Tanamachi, D. M., Scarpellino, L. \& Held, W. Initiation and limitation of Ly-49A NK cell receptor acquisition by T cell factor-1. J. Immunol. 171, 769-775 (2003). 
95. Held, W., Kunz, B., Lowin-Kropf, B., Wetering, M. \& Clevers, H. Clonal acquisition of the Ly49A NK cell receptor is dependent on the trans-acting factor TCF-1. Immunity 11, 433-442 (1999).

96. Yui, M. \& Rothenberg, E. Developmental gene networks: a triathlon on the course to T cell identity. Nat. Rev. Immunol. 14, 529-545 (2014).

97. Rothenberg, E., Ungerbäck, J. \& Champhekar, A. Forging T-lymphocyte identity: intersecting networks of transcriptional control. Adv. Immunol. 129, 109-174 (2016).

98. Cherrier, D., Serafini, N., Di \& Santo, J. Innate lymphoid cell development: a T cell perspective. Immunity 48, 1091-1103 (2018).

99. Laurenti, E. et al. The transcriptional architecture of early human hematopoiesis identifies multilevel control of lymphoid commitment. Nat. Immunol. 14, 756-763 (2013).

100. Kohn, L. A. et al. Lymphoid priming in human bone marrow begins before expression of CD10 with upregulation of L-selectin. Nat. Immunol. 13, 963-971 (2012).

101. Doulatov, S. et al. Revised map of the human progenitor hierarchy shows the origin of macrophages and dendritic cells in early lymphoid development. Nat. Immunol. 11, 585-593 (2010).

102. Adolfsson, J. et al. Upregulation of Flt3 expression within the bone marrow Lin(-) Sca1 $(+)$ c-kit(+) stem cell compartment is accompanied by loss of self-renewal capacity. Immunity 15, 659-669 (2001).

103. Adolfsson, J. et al. Identification of Flt3 + lympho-myeloid stem cells lacking erythro-megakaryocytic potential a revised road map for adult blood lineage commitment. Cell 121, 295-306 (2005).

104. Berthault, $C$. et al. Asynchronous lineage priming determines commitment to $\mathrm{T}$ cell and B cell lineages in fetal liver. Nat. Immunol. 18, 1139-1149 (2017).

105. Yoshida, $H$. et al. Expression of alpha(4)beta(7) integrin defines a distinct pathway of lymphoid progenitors committed to $T$ cells, fetal intestinal lymphotoxin producer, NK, and dendritic cells. J. Immunol. 167, 2511-2521 (2001).

106. Ramond, C. et al. Two waves of distinct hematopoietic progenitor cells colonize the fetal thymus. Nat. Immunol. 15, 27-35 (2014).

107. Elsaid, R. et al. A wave of bipotent T/ILC-restricted progenitors shapes the embryonic thymus microenvironment in a time-dependent manner. Blood 137, 1024-1036 (2020).

108. Koga, S. et al. Peripheral PDGFRagp38 mesenchymal cells support the differentiation of fetal liver-derived ILC2. J. Exp. Med. 215, 1609-1626 (2018).

109. Gasser, R. F. Atlas of human embryos. J. Anat. 120, 607 (1975).

110. O'Rahilly, R., Muller, F. \& Streeter, G. L. Developmental stages in human embryos: including a revision of streeter's horizons and a survey of the carnegie collection. Bulletin de l'Association des anatomistes. 637, 160 (1987).

111. Butler, A., Hoffman, P., Smibert, P., Papalexi, E. \& Satija, R. Integrating single-cell transcriptomic data across different conditions, technologies, and species. Nat. Biotechnol. 36, 411-420 (2018).

112. Wolf, F. A., Angerer, P. \& Theis, F. J. SCANPY: large-scale single-cell gene expression data analysis. Genome Biol. 19, 15 (2018).

113. Wolf, F. A. et al. PAGA: graph abstraction reconciles clustering with trajectory inference through a topology preserving map of single cells. Genome Biol. 20, 59 (2019).

114. Aibar, S. et al. SCENIC: single-cell regulatory network inference and clustering. Nat. Methods. 14, 1083-1086 (2017)

115. Velten, L. et al. Human haematopoietic stem cell lineage commitment is a continuous process. Nat. Cell. Biol. 19, 271-281 (2017).

116. Hou, S., Li, Z., Zheng, X., Gao, Y. \& Lan, Y. Embryonic endothelial evolution towards first hematopoietic stem cells revealed by single-cell transcriptomic and functional analyses. Cell Res. 30, 376-392 (2020).
117. Lange, M., Bergen, V., Klein, M., Setty, M. \& Theis, F. J. CellRank for directed singlecell fate mapping. https://www.biorxiv.org/content/10.1101/2020.10.19.345983v1 2020

118. Gulati, G. S. et al. Single-cell transcriptional diversity is a hallmark of developmental potential. Science 367, 405-411 (2020).

\section{ACKNOWLEDGEMENTS}

This study was supported by grants from the National Key R\&D Program of China (2020YFA0112400, 2019YFA0110200, 2017YFA0103401, 2016YFA0100601 and 2016YFA0502203), the National Natural Science Foundation of China (31425012, $31930054,31871173,81890991,82025002,81871232,81800102$ and 81900115$)$, the Program for Guangdong Introducing Innovative and Entrepreneurial Teams (2017ZT07S347), the Beijing Municipal Science \& Technology Commission (Z171100000417009 and Beijing Leading Talents Program Z171100001117159), the State Key Laboratory of Proteomics (SKLP-K202003) and the Key Research and Development Program of Guangdong Province (2019B020234002).

\section{AUTHOR CONTRIBUTIONS}

$\mathrm{BL}, \mathrm{YL}$ and $\mathrm{HH}$ designed the study. $\mathrm{HY}$ provided the samples. $\mathrm{CL}$ and $\mathrm{HZ}$ performed the sample preparation, the FACS and functional experiments with help from $Y Z$, ZBian, QX, JY, MZ, ZBai and YN. CL and HZ performed the scRNA-seq. LB, YZ, CL, and $\mathrm{HZ}$ collected and prepared the samples. $\mathrm{YG}$ performed the bioinformatics analysis with help from $\mathrm{JH}, \mathrm{ZL}$ and $\mathrm{JZ}$. CL, HZ, YG, YL, BL and $\mathrm{HH}$ wrote the manuscript.

\section{COMPETING INTERESTS}

The authors declare no competing interests.

\section{ADDITIONAL INFORMATION}

Supplementary information The online version contains supplementary material available at https://doi.org/10.1038/s41422-021-00529-2.

Correspondence and requests for materials should be addressed to A.W., Y.L., H.H. or B.L.

Reprints and permission information is available at http://www.nature.com/ reprints

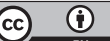

Open Access This article is licensed under a Creative Commons Attribution 4.0 International License, which permits use, sharing, adaptation, distribution and reproduction in any medium or format, as long as you give appropriate credit to the original author(s) and the source, provide a link to the Creative Commons license, and indicate if changes were made. The images or other third party material in this article are included in the article's Creative Commons license, unless indicated otherwise in a credit line to the material. If material is not included in the article's Creative Commons license and your intended use is not permitted by statutory regulation or exceeds the permitted use, you will need to obtain permission directly from the copyright holder. To view a copy of this license, visit http://creativecommons. org/licenses/by/4.0/.

(c) The Author(s) 2021 